\title{
Polymer Photocatalysts for Solar-to-Chemical Energy Conversion
}

\author{
Tanmay Banerjee, ${ }^{1}$ Filip Podjaski, ${ }^{1}$ Julia Kröger, ${ }^{1,2}$ Bishnu P. Biswal ${ }^{1}$ and Bettina V. Lotsch* ${ }^{1,2,3}$ \\ ${ }^{1}$ Max Planck Institute for Solid State Research, Heisenbergstraße 1, Stuttgart, Germany \\ ${ }^{2}$ Department of Chemistry, University of Munich (LMU), Butenandtstraße 5-13, München, Germany \\ ${ }^{3}$ E-conversion and Center for Nanoscience, Schellingstraße 4, München, Germany \\ * To whom correspondence is to be addressed - b.lotsch@fkf.mpg.de
}

\begin{abstract}
Solar-to-chemical energy conversion for the generation of high energy chemicals is one of the most viable, non-intermittent solutions to the present-day lookout for sustainable energy resources. Recently, organic polymeric photocatalysts have garnered significant attention in this field of research, which has long been dominated by inorganic semiconductors. Revisiting the lessons learnt from the latter, we reevaluate the fundamental concepts of photocatalysis in the context of the different classes of polymeric photocatalysts known to date, ranging from carbon nitrides and conjugated polymers, to covalent triazine frameworks and covalent organic frameworks. We then analyze the photophysical and physicochemical concepts that govern the photocatalytic performance of these materials, and thereby derive pertinent design principles and possible future research directions in this emerging field of "soft photocatalysis".
\end{abstract}

\section{Summary}

Organic polymer photocatalysts have garnered significant recent attention in solar-to-chemical energy conversion research. In this critical review, we analyze the fundamental concepts that govern the photocatalytic performance of these materials, and thereby derive pertinent design principles and possible future research directions in this emerging field of "soft photocatalysis".

\section{Introduction}

Fossil fuels are intrinsically unsustainable energy resources and continue to be depleted due to their overexploitation over the past few decades. From an environmental point of concern, the cost of combustion of fossil fuels is high and has resulted in an alarming rise in the atmospheric $\mathrm{CO}_{2}$ emission levels causing global warming and climate change. Photocatalysis as a discipline has evolved in response to these challenges, inspired by the concept of making solar fuels using nature as a blueprint, that is through artificial photosynthesis. 
The term photocatalysis implies a reaction that requires a catalyst and light simultaneously. This is distinct from sensitized photochemical reactions, where the 'catalyst' - also called the (photo)sensitizer - absorbs light and transfers energy to the reactant to generate its excited state and thus initiates a photochemical reaction at the excited state potential energy surface of the reactant without further participating in it. A photocatalyst on the other hand activates the reactant through a chemical reaction, such as an electron or atom transfer (and not through a physical step like energy transfer), and catalyzes an entirely ground state transformation. ${ }^{1-3}$ A true photocatalytic reaction is a thermodynamically favourable process $(\Delta \mathrm{G}<0)$; the photocatalyzed thermodynamically unfavourable process with $\Delta \mathrm{G}>0$ is a photosynthetic reaction, ${ }^{2,4}$ a differentiation not justly presented in most of the existing literature. For practical reasons however, we keep compliance with the common use of these terms in the literature for an easier understanding and better correlation.

The scope of photocatalytic reactions is huge. Photoredox catalysis - a rapidly growing subfield of photocatalysis - is particularly useful in organic chemistry as a means of overcoming the activation energy associated with a particular reaction and thereby exploiting alternative reaction pathways, as manifested in milder reaction conditions and shorter reaction sequences. ${ }^{5,6}$ Nevertheless, this review discusses the scope of such reactions solely in the context of the present day energy conundrum, focusing on photocatalytic reactions where light energy is transformed and stored as chemical energy - either in the form of solar fuels or other high value chemicals.

The seminal work of Honda and Fujishima in 1972 on photoelectrochemical water splitting using n-type semiconductor $\mathrm{TiO}_{2}$ electrodes laid the foundations of solar photocatalysis research. ${ }^{7}$ The following decades saw extensive research in this direction towards solar fuel production and environmental remediation using inorganic heterogeneous photocatalysts, primarily metal oxides and chalcogenides, owing to their activity and stability. ${ }^{8-10}$ The difficulties in tuning and adapting such inorganic photocatalysts led to the uprise of molecular homogeneous systems in the recent decades, however at the expense of stability and efficiency. ${ }^{11-13}$

The present review will discuss the development and advances in the field of 'soft photocatalysis' using organic polymeric photocatalysts, namely carbon nitrides, $\pi$-conjugated polymers, covalent triazine frameworks and covalent organic frameworks. ${ }^{14,15}$ Such polymer photocatalysts tend to combine the merits of homogeneous and heterogeneous photocatalysis, thus constituting a promising new class of nextgeneration photocatalysts. Such photocatalysts offer a vast scope for molecular-level design due to their molecular backbone and at the same time are much easier to tune through solution-phase chemistry. Being composed only of earth abundant and light elements, they are potentially cheaper than their inorganic semiconductor counterparts and considered relatively non-toxic. Furthermore, despite having a molecular backbone, polymeric photocatalysts retain the aforementioned robustness, stability, recoverability and scalability of inorganic semiconductor photocatalysts, unlike the (highly tunable) molecular homogeneous photocatalysts.

The present article does not intend to be a comprehensive collocation of all existing types of polymeric photocatalysts, nor does it give a detailed account of their photocatalytic performance. Instead, the review revisits and re-evaluates concepts innate to traditional semiconductor photocatalysts in order to materialize an understanding of the fundamental principles governing the photocatalytic processes in organic polymeric materials. It then presents a photophysical and physicochemical understanding of the role and the importance of the various material properties affecting these photocatalytic processes in the aforementioned 
organic polymeric materials, thus enabling identification of design principles and possible future research directions using such materials.

\section{Physicochemical foundations \\ Classical semiconductor photocatalysis}

Photocatalysis can be deconvoluted into four independent steps, which together define the overall photocatalytic efficiency. These steps are light absorption leading to exciton formation, charge carrier separation, their transport to the surface catalytic center, and the surface catalytic reaction with the reactant which most often occurs with the assistance of co-catalysts. ${ }^{16,17}$ The surface catalytic reaction is a chemical process that depends on the performance of the variable co-catalyst and is therefore not addressed at this stage. Light absorption as well as all subsequent steps assisting charge carrier percolation however severely depend on the photophysical and physicochemical properties of the light absorbing semiconductor material. It should be noted that the 'photocatalyst' is this semiconductor material or an ensemble thereof where the actual electrocatalyst is attached to a light absorbing material. However, the light absorbing material can also be colloquially called the photosensitizer depending on the scientific community.

The amount of light that can be absorbed depends on the band gap energy, the type of the band gap (direct or indirect for inorganic semiconductors, or HOMO-LUMO gap for organic materials) and the material's absorbance that together determine the fraction of the incident light that is absorbed and hence, the generation rate of excited charge carriers. External low band gap photosensitizers such as organic or metal complex dyes may increase light absorption, as in dye sensitized solar cells (DSSCs). ${ }^{18}$ The absolute band positions on the other hand determine the thermodynamic driving force for the surface catalytic reaction and need to be taken into account for driving the particular reaction of interest, for example, water splitting or $\mathrm{CO}_{2}$ photoreduction, the energetic requirements of which are shown in Figure 1. ${ }^{17,19,20}$

$<$ Figure 1>

After light absorption, the generated electron $\left(\mathrm{e}^{-}\right)-$hole $\left(\mathrm{h}^{+}\right)$pairs relax into excitons, which need to be separated to prevent carrier recombination. Hence, the exciton binding energy $\left(\mathrm{Ex}_{\mathrm{BE}}\right)$ and the exciton diffusion length are crucial parameters which are heavily affected by the particle size and morphology in dispersed systems. The free charge carriers $\left(\mathrm{e}^{-}\right.$and $\left.\mathrm{h}^{+}\right)$can then move within their diffusion length towards the surface and the catalytic center, often assisted by band bending or the formation of heterojunctions with other semiconductors.

Depending on the amount of (intrinsic) doping, which defines the number of mobile electrons and/or holes in the material, a space charge layer is naturally formed at the interface with the surrounding electrolyte as a consequence of Fermi level $\left(\mathrm{E}_{\mathrm{F}}\right)$ alignment between the photocatalyst and the electrolyte, generating a surface band bending which provides the driving force for charge carrier transport (Figure 2). ${ }^{21,22}$ For ntype semiconductors with electrons as the majority carriers, an upward band bending is typically observed, assisting minority carrier (here, holes) transport towards the surface and driving electrons towards the bulk. p-type semiconductors on the other hand show the opposite behavior. Hence, one doped semiconductor alone is usually able to drive only one (anodic or cathodic) surface reaction, unless the free charges are photogenerated close to the surface and/or tunnel through the energetic barriers. Besides the intrinsic doping of the semiconductor, surface states and traps can contribute to the band bending and cause Fermi level 
pinning affecting charge transport and recombination at the interface. ${ }^{17,23,24}$ Knowledge of the bulk band structure of the photocatalyst in vacuum is therefore not deemed sufficient to describe the energetic driving force for a reaction in aqueous conditions, nor charge separation and transport towards the surface. This is because junctions are formed at the interfaces (due to the formation of a space charge layer or due to (surface) trap states) that create a built-in potential via the space charge layer and thereby shift the bulk band positions with respect to the surface, hence modifying the thermodynamics of charge carriers created in the bulk. ${ }^{17,21}$ Furthermore, the energetic position of surface related band edges depends on the $\mathrm{pH}$ of the aqueous reaction medium. ${ }^{25,26}$

\section{Organic semiconductor photocatalysts}

It is rather challenging to link the aforementioned processes occurring within inorganic photocatalysts to organic semiconductor photocatalysts in a rigorous manner, since the extraction of intrinsic parameters influencing the efficiency of the overall photocatalytic process is often difficult in practice. Examples include the band position of the material in the electrolyte, $\mathrm{Ex}_{\mathrm{BE}}$, exciton diffusion length, the bulk and grain boundary conductivity, the concentration of defects and their energetic levels, or the amount of free charge carriers (doping level) and hence the amount of band bending. This is especially so because the measurement of these intrinsic material parameters requires either large homogeneous crystals of high purity, or well-defined thin films, which is challenging to achieve for highly insoluble organic photocatalysts with 2D or 3D network topologies. Furthermore, the often large structural units render the creation of realistic theoretical models and their comparison with experimental data challenging. Hence, extraction of photophysical parameters requires sophisticated methods and instrumentation. ${ }^{15,25,27}$ Without the knowledge of effective semiconductor properties, such as the number of free charge carriers, the effective masses, trap densities and distributions, and charge carrier diffusion lengths, it is thus extremely challenging to address the real bottleneck in the overall photocatalytic process. Hence, descriptions and measurements of photophysical properties of organic particulate photocatalysts are often based on assumptions and, thus, oversimplified. A more detailed discussion of the differences and commonalities between inorganic and organic semiconductor photocatalysts is presented below.

$<$ Figure 2>

Charge carrier separation. Inorganic photocatalysts have fairly high charge carrier mobilities, ${ }^{28,29}$ described by low effective masses $m^{*}\left(1 / \mathrm{m}^{*}=1 / \mathrm{m}_{\mathrm{e}}^{*}+1 / \mathrm{m}_{\mathrm{h}}{ }^{*}\right)$ due to band transport and relatively high dielectric constants. As such, Ex $x_{\mathrm{BE}}$ can be described by the Wannier-Mott model: ${ }^{16}$

$\mathrm{Ex}_{\mathrm{BE}}=\mathrm{E}_{\mathrm{H}} \cdot \mathrm{m} * \varepsilon_{\mathrm{r}}^{2}$,

where $\mathrm{E}_{\mathrm{H}}$ is the energy of the $1 \mathrm{~s}$ orbital of hydrogen $(-13.6 \mathrm{eV})$ and $\varepsilon_{\mathrm{r}}$ is the relative permittivity or dielectric constant. Since $\mathrm{Ex}_{\mathrm{BE}}$ is often low $(\sim 10 \mathrm{meV})$ in inorganic systems, charge separation is relatively easy and often of the order of the thermal energy, $k \mathrm{~T}$.

Organic photocatalysts on the other hand have high $\mathrm{Ex}_{\mathrm{BE}}$ of the order of hundreds of meV up to $\sim 1 \mathrm{eV},{ }^{30-}$ 32 and are rather described by the Frenkel model that assumes strong exciton binding and small exciton Bohr radii with no extended separation of $\mathrm{e}^{-}$and $\mathrm{h}^{+}$(Figure $2 \mathrm{~b}$ ). Note that as mentioned above, the experimental extraction of $\mathrm{Ex}_{\mathrm{BE}}$ is difficult and thus its determination is typically based on computational methods. ${ }^{33-36}$ The high $\mathrm{Ex}_{\mathrm{BE}}$ can be rationalized by considering localized electronic states, akin to spatially 
separated HOMO and LUMO orbitals in the molecular picture, rather than extended valence and conduction bands, as well as a generally low dielectric constant. ${ }^{37}$ The strong interaction of the exciton with the molecular backbone often manifests as polaronic transport, ${ }^{38-41}$ while the low $\varepsilon_{\mathrm{r}}$ hinders charge screening, leading to strong binding of $\mathrm{e}^{-}$and $\mathrm{h}^{+}$. As a consequence, low exciton diffusion lengths and high charge carrier recombination rates result, thus limiting the quantum efficiency of organic based materials. ${ }^{42}$

However, donor-acceptor systems which stabilize the excitonic carriers in a charge transfer state, ${ }^{43}$ the formation of defects in the bulk or at the surface, ${ }^{44,45}$ the formation of semiconductor heterojunctions, ${ }^{46,47}$ and the interaction with co-catalysts on the surface, ${ }^{15,48}$ can help drive the separation of excitons. Alternatively, the binding energy of excitons can be reduced by varying the polymer chain length or increasing their dimensionality. ${ }^{32,36,49}$ A low intrinsic exciton diffusion length mandates exciton separation close to its generation to prevent unproductive recombination before the carriers can be extracted. As a result, photocatalytic experiments with such materials are usually performed with small, well-dispersed particles or with high surface area materials, or highly porous materials with increased solution permeability. ${ }^{15}$ Porosity, on the other hand, may decrease the effective dielectric constant of the material. However, if the pores of a photocatalyst are filled with water, the relatively high dielectric constant of water $(\sim 80)$ compared to the solid may contribute to the effective dielectric property of the material and assist charge separation. Further, sacrificial agents are frequently employed to artificially separate the strongly bound excitons by quenching the photogenerated electron or hole via kinetically facile one-electron processes with a high thermodynamic driving force, thus bypassing the intrinsic material limitation. The high surface area and the semi-crystallinity or amorphous nature of organic materials can manifest in a high density of defects, which may be beneficial for exciton dissociation, but on the other hand, can also enhance charge recombination. ${ }^{27,39,50-52}$ The defects can also act as catalytic centers and even facilitate an in-situ attachment of co-catalysts, thereby providing energetic sinks for photogenerated charge carriers (vide infra) ${ }^{45,53}$ The aforementioned strong exciton binding in organic polymeric photocatalysts, and the defects which can also act as charge carrier traps, may further give rise to energy levels in the electronic band gap (Figure 2c), thus enabling light absorption (and hence possibly enhanced photocatalytic activity) at energies below the true electronic band gap between the top of the valence band and the bottom of the conduction band. . $^{17,52,54}$

Charge carrier transport. As outlined above, in inorganic semiconductors, the separation of excitons occurs rather easily and hence, the transport of unbound $\mathrm{e}^{-}$and $\mathrm{h}^{+}$occurs in their respective bands. The conductivity, $\sigma$ is described by the product of the concentration of dopant and hence, the amount of majority carriers, $\mathrm{N}_{\mathrm{D}}$, and the charge carrier mobility, $\mu:^{.5}$

$\sigma=\mathrm{e} \cdot \mathrm{N}_{\mathrm{D}} \cdot \mu$

with $e$ being the elementary charge. The mobility and hence, the transport is limited by scattering events stemming from defects or grain boundaries with a characteristic collision lifetime $\tau$, as described by the Drude model: ${ }^{55}$

$\mu=\mathrm{e} \cdot \tau / \mathrm{m}^{*}$

While deep traps severely impede charge carrier migration, shallow traps have only minor effects on the conductivity. ${ }^{56,57}$ As a result of the typically high crystallinity, tuning of defects and the concentration of free charge carriers by doping, bulk charge transfer properties can be predicted and adjusted to obtain a relatively long-range conductivity in inorganic systems. 
On the other hand, for organic polymeric photocatalysts like carbon nitrides, covalent triazine frameworks and covalent organic frameworks this is not necessarily the case. Charge transport mainly occurs via hopping rather than band-like transport - due to extended $\pi$-conjugation or facile formation of polarons. ${ }^{38-}$ ${ }^{41,58}$ The measurement of the intrinsic bulk charge transfer properties is challenging and not easily addressable with computational methods either. This is due to structural defects lying deep in the band gap arising from the heterogeneity of the chain length and its branching in the polymeric material, ${ }^{52}$ as well as typically small crystallite sizes coupled with large grain boundary resistance. The lack of polarizability (low $\varepsilon_{\mathrm{r}}$ ) coupled to low concentrations of mobile charge carriers and high energy barriers for charge transfer to/from the electrolyte (equivalent to a high energy penalty for oxidation or reduction of chemical bonds) also has a severe influence on possible band bending. As such, true n- or p-type conductivity in classical terms is questionable in organic semiconductor photocatalysts and, hence, the viability of assumptions based on the existence of a space charge region. It has been shown that for conductive organic semiconductors, only the free charge carriers responsible for effective charge carrier mobility actually contribute to the formation of space charge layers, which results in band bending at an interface ${ }^{59}$ For such polymers, it has also been shown that band bending on the surface actually depends on the film thickness. Thus, at low concentrations of mobile charge carriers, which is considered the default situation in the majority of polymer photocatalysts, the formation of a space charge layer with significant extension is questionable. For small particles, this space charge layer is even more difficult to access since the width of the layer is inversely proportional to the free charge carrier concentration. ${ }^{60,61}$ This has implications with regard to the Mott-Schottky analysis performed to obtain band positions in organic materials and can lead to erroneous interpretations. ${ }^{62-64}$ For such measurements, special care needs to be taken in revealing the actual origin of the measured capacitance, that is whether it stems from the space charge layer only, or if it is influenced by the Helmholtz layer, by a chemical capacitance due to ionic interactions, or by a diffusion capacitance originating from the injection of charge carriers at a contact or interface which can play a dominant role in low doped materials ${ }^{60,62,63}$ Furthermore, Mott-Schottky analysis should not only be used for the extraction of a possible flat band potential from the intercept, but also the free charge carrier density and dielectric constant (which can both be extracted from the slope) should be compared to other measurements and verified to be in a reasonable range.

The near absence of a space charge layer for most organic semiconductors has severe implications on photocatalysis. In case of band bending as in inorganic systems (Figure 2d), excited charge carriers need to reach the depletion or accumulation region only (n- or p-type materials, respectively) as the band bending separates and transports them further. Without such band bending, that is with rather flat bands at the interface, charge carriers need to reach the interface within the very limited diffusion length. Charge separation in organic, non-conductive photocatalysts should thus occur near the interface, possibly assisted by tunneling into lower energetic states as provided by surface defects or metal nanoparticles (Figure 2e). Charge separation at the interface can also be assisted by molecular level tuning of the intrinsic polarity of the organic material as discussed in a later section.

In spite of all these challenges, organic polymers have undergone an impressive development in their ability to drive photocatalytic reactions. Enabled by the atomic level tunability of their backbone - a unique feature compared to inorganic systems - both classically employed structural and electronic optimizations by rational backbone engineering as well as identification and targeted introduction of active sites have been used to address and optimize light harvesting, charge separation and transport properties. We discuss some instances exemplifying these aspects for the different classes of organic polymer photocatalysts (Figure 3 ) 
- carbon nitrides, $\pi$-conjugated polymers, covalent triazine frameworks and covalent organic frameworks in the sections below.

$<$ Figure 3>

\section{Carbon nitrides}

\section{Absorption, charge separation and transport}

Carbon nitrides have evolved as powerful photocatalysts primarily due to their oxidative and reductive power, that is appropriate energy levels for proton reduction, water oxidation and $\mathrm{CO}_{2}$ reduction, their visible light activation, high chemical stability, and their straightforward synthesis from inexpensive precursors. ${ }^{14,65-70}$ In fact, the ascent of organic polymer photocatalysis can be linked to the seminal work by Antonietti, Domen and co-workers in $2009,{ }^{71}$ demonstrating water reduction and oxidation using a heptazine-based polymer photocatalyst known as melon. ${ }^{72,73}$ Often simplistically termed graphitic carbon nitride or $\mathrm{g}_{-} \mathrm{C}_{3} \mathrm{~N}_{4}$, melon is composed of heptazine units linked to each other via secondary amine bridges to form one-dimensional chains (Figure 3a). ${ }^{73,74}$ The chains are hydrogen-bonded forming 2D layers, which are stacked in the third direction by $\pi-\pi$ interactions.

While melon-type materials are obtained by classical solid-state synthesis at temperatures $>500{ }^{\circ} \mathrm{C}$, synthesis under ionothermal conditions has led to the development of "true" 2D crystalline carbon nitrides. ${ }^{65,75,76}$ Here, the salt melt simultaneously acts as a high-temperature flux and the constituent ions as structure directing agents, leading to a higher degree of condensation and significantly enhanced structural perfection. Two members of this class of carbon nitrides have been structurally characterized: lithium halide intercalated poly(triazine imide) $\left(\mathrm{PTI}^{\mathrm{Li}}{ }^{+} \mathrm{X}^{-}\right),{ }^{75,77-80}$ and poly(heptazine imide) (M-PHI) where $\mathrm{M}$ is either a proton or metal counter ion, ${ }^{76,81-84}$ both of which consist of imide-bridged triazine or heptazine building units forming a graphite-like two-dimensional in-plane structure with regular structural voids (Figure 3b). The crystalline sheets are then stacked in the third direction with the stacking polytype depending on the pore content. ${ }^{76}$ The intercalation of ions in the crystal structure of carbon nitrides has been suggested to have a significant influence on the photocatalytic activity; it has been argued that coordinating metal ions assist separation of photogenerated charge carriers and also improve the electrical conductivity of the material. ${ }^{83,85}$ Interaction of the valence and the conduction band states with the intercalated ions in $\mathrm{PTI} / \mathrm{Li}^{+} \mathrm{Cl}^{-}$has further been shown to reduce its band gap compared to polymeric melon. ${ }^{86}$ In general, the different types of carbon nitrides, - melon, PTI and PHI - have distinct optical and photocatalytic properties. In their native form, 2D PTI and PHI outperform 1D melon in terms of photocatalytic hydrogen evolution (Table 1) in the presence of metallic platinum as the co-catalyst and a sacrificial electron donor (vide infra) ${ }^{75,76}$ It has to be noted however that melon has the most negative conduction band among these carbon nitrides and thus the highest thermodynamic driving force for the hydrogen evolution reaction (HER). The opposite trend in the observed hydrogen evolution performance thus points to multiple including kinetic - factors affecting this reaction.

$<$ Table 1>

Light absorption, charge separation and transfer in carbon nitrides is severely influenced by structural properties and real structure effects, including crystallinity, stacking distances, and defects (Figure 4a). In 
melon-type carbon nitrides, an improved interlayer exciton dissociation and lateral charge transport was observed for smaller stacking distances and higher crystallinity. ${ }^{35}$ An increase in crystallinity has also been argued to be responsible for increased non-radiative relaxation pathways in PHI materials, reflected in the progressive decrease of fluorescence lifetimes, which has been associated with possible charge transfer to favorable surface states. ${ }^{84}$ However, this trend was not found to be reflected in the $\mathrm{H}_{2}$ evolution activity of the material. The increased structural order and the 2D topology of carbon nitrides can further result in an increase in $\pi$-conjugation which could lower the band gap, leading to enhanced light absorption. ${ }^{75}$ Regarding the band positions, PHI represents an intriguing case where the valence band potential is highly positive (K-PHI: +2.22 vs. NHE), thereby rendering the photoexcited holes very strong oxidants. ${ }^{87-91}$ The negative surface charge of the PHI backbone can further enhance the stability of the photocatalyst dispersion by interparticle repulsion, thereby maximizing light exposure of the catalyst surface and its accessibility to the reactants during photocatalysis. ${ }^{84,92}$

Understanding the fundamental photophysical processes in carbon nitride photocatalysis is challenging, ${ }^{15,39}$ as measurements are often impeded by the difficulties in producing samples of near-optical quality. Photoluminescence (PL) spectroscopy has been extensively used to study the photophysics of melon and early results pointed to the generation of singlet Frenkel excitons highly confined to the heptazine units upon photoexcitation owing to the lack of significant conjugation within the 1D strand. ${ }^{93-95}$ This gives rise to high $\operatorname{Ex}_{\mathrm{BE}}(\sim 1 \mathrm{eV})^{34,96}$ making exciton dissociation difficult. The material was thus suggested to be an optical quasi-monomer. ${ }^{93}$ It was also observed that the transition energies and the excitonic coupling depend on the interplanar distance between the heptazine units. Based on a non-exponential decay of the PL signal, the exciton transport was found to be inter- rather than intraplanar (Figure 4b), as opposed to graphene and possibly linked to the 1D character of melon. Transient absorption spectroscopic studies on melon-like carbon nitride polymers suggested that the singlet excitons created in the heptazine building blocks after photoexcitation dissociate into singlet polaron pairs in adjacent layers in $<200 \mathrm{fs} .{ }^{38}$ These polaron pairs further dissociate and diffuse one dimensionally along the stacking direction in a Brownian motion mediated by an overlap of the molecular orbitals. On the contrary, since charge separation is suppressed in few layer carbon nitrides due to the high electronic barriers for intralayer transport, singlet excitons persist in such materials and produce high PL yields. ${ }^{97}$ It was further envisaged that with an increase in the hopping rate and mobility of polarons together with the suppression of exciton recombination due to spin thermalization the photocatalytic activity of such melon-like materials can increase as the chances of the charge carriers to reach the active sites on the surface of the material increases. It is however important to note that delayed radiative excitonic recombination due to triplet-triplet annihilation could also constitute a prominent excited state depopulation pathway for such materials. ${ }^{98}$ Intriguingly, concerning the excited state landscape of carbon nitrides, a $S_{1} / T_{1}$ inversion in the excited state of a heptazine derivative and linearly connected small oligomers of melem was recently suggested, based on calculations and supporting timeresolved photophysical experiments. ${ }^{99}$ This implied that the lowest excited state in graphitic carbon nitride materials could be a singlet state and not a triplet state. Further photophysical investigation of carbon nitride polymers with regard to this effect will however be essential in order to develop an understanding and exploit the implications in photocatalytic applications.

The anisotropy in charge transfer was further confirmed for $\mathrm{LiCl}$ containing $2 \mathrm{D} \mathrm{PTI},{ }^{27}$ in which the electrical conductivity between the layers was observed to be 65 times higher than within one layer $(1.0 \times$ $10^{-4} \mathrm{~S} \mathrm{~m}^{-1}$ vs. $1.6 \times 10^{-6} \mathrm{~S} \mathrm{~m}^{-1}$ ). The diffusive hopping mobility of polarons (which governs the charge transport in the material) obtained by transient absorption spectroscopy was in the range of $0.8-2.4$ $\mathrm{cm}^{2} \mathrm{~V}^{-1} \mathrm{~s}^{-1}$ depending on the dimensionality of polaron diffusion (3D or 1D). This is in stark contrast to the 
3 or 4 orders of magnitude larger in-plane conductivity over out-of-plane conductivity in other layered materials such as $\mathrm{MoS}_{2},{ }^{100}$ and graphite ${ }^{101}$. Unfortunately, the dominant type of charge carriers responsible for the reported conductivity values and the carrier concentrations were not identified.

In principle, a high concentration of either stabilized or free, long-lived charges is desired for high photocatalytic activity as the reaction probability is increased. PHI represents a unique case where ultra long-lived electrons can be trapped on the material (storage capacities upto $92 \mathrm{C} \mathrm{g}^{-1}$ ) after photoreduction in the presence of a sacrificial electron donor. ${ }^{87,102,103}$ The trapped electrons can then be de-trapped on demand and consumed for the generation of hydrogen or other fuels by addition of Pt colloid in the dark after a time delay of hours. ${ }^{102}$ This process, called 'dark photocatalysis', thus separates the light (that is, light absorption) from the dark process (that is, catalysis) of photocatalysis, akin to natural photosynthesis, pointing to a strategy towards overcoming the intermittency of solar irradiation. This unusual charge storage capability has also been used to create a solar battery photoanode with PHI. ${ }^{104}$ Recently it was shown that upon excessive charge accumulation on the material, recombination of the photogenerated charge carriers becomes more likely, hence suppressing light-induced free charge carrier generation effectively. ${ }^{105}$ Studies of charge carrier dynamics by transient absorption spectroscopy showed a 400-fold increased recombination rate in the presence of hole scavengers, caused by long-lived electron accumulation after hole extraction. To circumvent this problem redox mediators were used to "outsource" the electrons and keep them from building up on PHI. A high charge carrier concentration is therefore beneficial for photocatalysis, as long as it does not promote carrier recombination. Maintaining a balanced electron and hole extraction kinetics is thus important for optimizing photocatalytic efficiency. It is also important to note that after quenching of the photoexcited holes by electron donors, reversible hole transfer from the oxidized donors chemisorbed on the catalyst surface can lead to unwanted charge recombination in the material, thus calling for an effective design strategy that prevents the oxidation products of the donor from accumulating at the surface of the photoabsorber. ${ }^{106}$

The morphology, represented by the micro- and nanostructure of carbon nitrides, including their porosity and surface area, is another structural aspect of profound importance for photocatalysis. Several methodologies including exfoliation, ${ }^{107-111}$ templating, ${ }^{112-115}$ and supramolecular preorganization, ${ }^{116,117}$ have been adopted to improve the photocatalytic activity of carbon nitrides ${ }^{66,67}$ Exfoliation of melon-type or crystalline PTI carbon nitride materials into monolayer or multilayer nanosheets represents a top-down strategy to study the relationship between morphological features and photocatalytic activity. In general, the as-produced nanosheets can possess large planar extensions $(0.5$ to several $\mu \mathrm{m}),{ }^{108}$ a significantly larger surface area and thus better exposure of "active sites" than their bulk counterparts. In addition, they can elicit longer lifetimes of photogenerated charge carriers and more efficient charge transfer properties than bulk materials, and this has been argued to be due to quantum confinement effects or possibly due to modified electron-phonon interactions in the material which can affect its conductivity. ${ }^{111,118}$ As a result of one or a combination of these factors, higher photocatalytic activities of the respective nanosheet materials have been reported compared to the bulk material. ${ }^{107,108,119}$ Another common approach to enhance the catalytic activity of carbon nitrides is by means of surface area engineering through the generation of porosity. Mesoporosity can be achieved by both soft and hard templating, ${ }^{114}$ for example by nanocasting using silica templates followed by chemical etching of the template. ${ }^{12,113}$ Suitably architectured mesoporous carbon nitride materials can have large surface areas $\left(>400 \mathrm{~m}^{2} \mathrm{~g}^{-1}\right){ }^{120}$ and accessible open meso- and macropores which can facilitate mass transfer. In such porous architectures favouring short charge carrier travel distances, charge separation and collection can thus be facilitated. ${ }^{112}$ Also, light 
harvesting may be enhanced through optimized scattering, thus leading to higher apparent quantum efficiencies. $^{112,113}$

<Figure 4>

\section{Defect and disorder engineering}

Not only higher crystallinity but also amorphization has been suggested to affect the charge separation properties in carbon nitrides in a beneficial way. Amorphization of melon - wherein the long range atomic order, mainly the intralayer hydrogen-bond interactions, are disrupted and a significant amount of dangling amine moieties removed - in fact renders it more active in HER as compared to pristine melon. ${ }^{121}$ A charge trapping model was recently developed in the transient absorption and time resolved PL study of carrier dynamics in melon-type carbon nitrides (Figure 5b). ${ }^{52}$ In this model the emissive states formed initially upon photoexcitation are proposed to be in thermal equilibrium with trapped charge separated states, wherein the population ratio is governed by the Boltzmann distribution. Analysis of their data based on this model revealed significant charge trapping in carbon nitrides with an exponential tail of trap states extending up to $1.5 \mathrm{eV}$ into the band gap which can accumulate deeply trapped electrons with lifetimes $\geq 1$ $\mu$ s. This corresponds to significant energy loss and consequently leads to a reduction in the driving force for charge transfer on $\mu$ s time scales and longer. The lower photoactivity of carbon nitride materials prepared at low temperatures, which have a higher density of defect sites - possibly amine moieties - due to less complete polymerization, was observed to be due to a higher population of deeply trapped electrons. Density functional theory (DFT) calculations on carbon nitride materials with typical defect functionalities suggested that the defects can indeed stabilize charge separated states relative to the emissive states by hundreds of $\mathrm{meV}$. Understanding the exact role of the defect sites in charge transfer steps during photocatalysis is thus crucial (vide infra) as such defects have also been suggested to be responsible for charge separation within the material rather than at the interface with the solvent.

\section{$<$ Figure 5>}

The nature of defects and their role in photocatalysis is particularly ambiguous in carbon nitrides, as can be seen by the many conflicting reports on the impact of "amine defects" on the photocatalytic activity (vide supra). For example, it was observed that the photocatalytic hydrogen evolution activity of oligomers of melem (Table 1), which naturally contain a larger number of primary amine sites, was as much as six times higher than that of polymeric melon and in fact, the activity of the oligomers increased with a decrease in chain length, albeit until a certain length. ${ }^{122}$ Faster kinetics of interfacial charge transfer was invoked as the most likely reason and indeed it was shown, based on DFT calculations, that the relative LUMO orbital coefficients on the amine groups, which possibly act as the reducing sites for HER, decreased progressively with increase in oligomer chain length, while the relative HOMO coefficients on the tertiary heptazine nitrogen atoms across oligomers of different lengths were largely unaltered. In addition, the amine groups are likely better exposed in the oligomers than in the polymer due to stacking disorder and conformational flexibility in the former. The modulation of kinetics of charge transfer via such structural amine groups was suggested to be either due to better coordination to the platinum centers for efficient electron transfer (Figure 5a), or due to amine-mediated proton-coupled electron transfer. The higher photocatalytic activity of the amorphous PTI polymer over its crystalline counterpart can likely be attributed to similar reasons. ${ }^{75}$ The importance of chain terminations in carbon nitride materials as photocatalytic centers and thereby the 
usefulness of oligomeric and/or molecular species in improving photocatalytic activity was further highlighted in a recent transient absorption spectroscopic study. ${ }^{123} \mathrm{In}$ an infusion of bulk-g- $\mathrm{C}_{3} \mathrm{~N}_{4}$ with exfoliated $\mathrm{g}-\mathrm{C}_{3} \mathrm{~N}_{4}$, a cooperative cascade effect was observed wherein charges are generated by exciton dissociation on the former followed by electron transfer to the latter in an almost diffusion controlled molecular interaction-like process. The exfoliated carbon nitride, which may not sustain long-lived charge separation itself, was assumed to have highly active catalytic sites, thus doubling the photocatalytic HER activity of bulk-g- $\mathrm{C}_{3} \mathrm{~N}_{4}$ (Table 1 ) in conjunction.

Defects in general and vacancy defects in particular can play an important role in modifying the electronic structure and charge transport properties of carbon nitride photocatalysts by introducing additional energy levels within the optical bandgap and/or acting as reactive sites. ${ }^{66,67,124-126}$ Nitrogen defects and vacancies for example, which act as trap sites, have been argued to decrease the radiative recombination of electrons and holes in $\mathrm{g}_{-} \mathrm{C}_{3} \mathrm{~N}_{4}$ and enhance photoexcited charge separation, thereby improving the photocatalytic activity of the material. ${ }^{127,128}$ However, neither the exact nature of such (high energy) structural defects nor the mobility of the charge carriers in these defect states is typically evaluated in such studies, which makes the correlation, let alone causation, with the observed catalytic activities highly speculative. Using time resolved PL and positron annihilation spectroscopy, it was shown that the trapped charge carriers in such defects states - composed mainly of uncondensed amine moieties or nitrogen vacancies - are mobile and can jump between the states to recombine with the photoexcited holes. ${ }^{129}$ Time resolved PL further revealed a significantly higher lifetime of the defect-bound exciton compared to the free exciton. Defects have also been deemed important for $\mathrm{CO}_{2}$ activation and photoreduction. ${ }^{130-132}$

For inorganic systems, elemental doping in controlled quantities adds mobile charge carriers with energies near the valence or conduction bands. For organic systems, "doping" usually refers to the substitution or addition of atoms or ions in the polymer backbone which, besides creating an often disregarded, significant amount of defect states, fundamentally modifies the backbone and hence can affect the HOMO and LUMO energies as well as more subtle structural details such as the conjugation length and particle size. Furthermore, the type and location of dopants is often not understood well. Nevertheless, this approach has been used extensively to modify the band gap in order to optimize light absorption in carbon nitride photocatalysts (Figure 4a). ${ }^{66,133}$ Also, elemental doping in such materials has been reported to improve charge separation and transport properties. ${ }^{134,135}$ In light of the importance of this strategy for improving the photocatalytic activity of carbon nitrides, it remains crucial to arrive at a deeper understanding of the structural implications of doping and its photophysical signature for the rational design of improved carbon nitride photocatalysts.

In summary, it must be noted that defects may or may not be beneficial for photocatalysis and this depends on the particular type of defect as well as the type of carbon nitride material used. Thus, generalizations must be taken with care and detailed investigation into the accompanying structural changes and the role of defects in influencing material properties like charge separation and transport are best carried out for every material individually.

\section{$\pi$-conjugated organic polymers}

\section{Optoelectronic engineering}

Carbon nitrides are composed strictly of alternating carbon and nitrogen atoms in the form of triazine or heptazine units and exclusively connected via imide bridges. As a result of the high-temperature synthesis of carbon nitrides and the resulting inertness of their backbone, the choice of building blocks and 
possibilities of functional group engineering is very limited, making fine tuning of the properties of carbon nitrides challenging. While much can be learnt from carbon nitride photocatalysis due to their excellent performance and robustness, a finer molecular level tuning and control of properties is needed to further our understanding of structure-property-function relationships in heterogenous organic photocatalysts.

$\pi$-conjugated polymer catalysts mark a significant step towards this goal. The advent of solar fuel photocatalysis using $\pi$-conjugated polymers dates back to 1985 with the demonstration of photocatalytic HER using poly(phenylene). ${ }^{136}$ About then, a number of linear poly(phenylene) type polymers had been explored and were found modestly active under UV irradiation (Table 2). ${ }^{137,138}$ The field has seen considerable growth in the past few years, ${ }^{14,15,139-149}$ with the pioneering work of Cooper and co-workers who started defining structure-property-activity relationships in the photoactivity of such polymers. As compared to the well-known organic polymers used in photovoltaics, ${ }^{150}$ these polymers are typically insoluble. Broadly, there are two classes of such polymers - the non-porous linear and the conjugated microporous polymers. ${ }^{139-141}$ As the name suggests, such photocatalysts are conjugated materials and it is possible to tune their optical gap (Figure 6a) by varying the oligomer length - or the degree of polymerization - thereby leading to a variation in the amount of light absorbed. ${ }^{151}$ The modularity of this photocatalyst platform enables the application of fundamental molecular photochemical principles to improve performance. For example, incorporating planar linkers enables a higher degree of conjugation and again a decreased optical gap and/or longer charge carrier lifetimes which contribute to improved photocatalytic performance. ${ }^{152,153}$ In fact, in donor-acceptor heterojunctions, it was observed that conjugation also allows for decreased Coulomb binding energy for exciton dissociation, improved charge carrier delocalization and mobility. ${ }^{154,155}$ It was further observed that conformational disorder reduces the mobility of charge carriers to the conjugation length. Increased carrier mobility was also achieved by planarization in triarylamine based conjugated polymers. ${ }^{156}$

\section{$<$ Figure 6>}

As mentioned before, photocatalytic activity is a compound product of the properties of the material and its interaction with the reactants - the latter an outcome of its composition and its microstructure. In a series of 1,4-phenylene and 2,5-thiophene linear co-polymers, it was observed using (TD)-DFT calculations that the adiabatic $\mathrm{Ex}_{\mathrm{BE}}$, as well as the driving force for proton reduction and oxidation of sacrificial electron donors decreased significantly with increased thiophene content. ${ }^{151}$ Thus, while increased thiophene content also resulted in a progressively smaller optical gap, the photocatalytic hydrogen evolution activity is maximum where the increased absorption of light compensates for the reduction in driving force for proton reduction and oxidation of sacrificial electron donors. Importantly, it was also observed that the exact composition of the polymer chain was crucial in determining the final photocatalytic hydrogen evolution activity and with an increase in the degree of segregation of the individual thiophene units, the driving force as well as the optical gap decreases because of localization of the singlet exciton and the separated charges on the thiophene rich segments of the polymer.

Using a similar copolymerization approach, a systematic and continuous control over the optical gap of pyrene based conjugated microporous polymers has been reported to be achieved by progressively increasing the content of pyrene. ${ }^{157,158}$ Note that contributions from pyrene dominate the low-energy excitations in the material. In this study, a steady increase in the hydrogen evolution activity of the polymers was observed with a decrease in the optical gap down to $2.33 \mathrm{eV}$ for the polymer CP-CMP10, after which 
the activity dropped (Figure 6b, Table 2). This was speculated to be due to detrimental electron-hole recombination or because of some incumbent kinetic barrier for proton reduction with increasing pyrene content.

With regard to the composition of organic polymer photocatalysts in general, it is important to note that $m e t a$-linkages limit the effective $\pi$-conjugation length in the framework and thus light absorption (Figure 6a), which is a crucial factor determining photocatalytic activity. ${ }^{159}$ The limited $\pi$-conjugation in such systems has also been reported to be detrimental for efficient exciton migration. ${ }^{160}$ Meta linkages further lead to diminished photoactivity by preventing achievement of high molecular weights. ${ }^{161}$

\section{Charge transport and catalysis}

As mentioned in a preceding section, the exciton diffusion lengths in typical conjugated polymers is very small, only around 5-20 nm, ${ }^{162}$ and thus in polymers synthesized in bulk most of the photogenerated excitons in the volume are not expected to reach the surface and participate in reactions. Thus, small dispersed particles are better for photocatalysis with such materials. ${ }^{163}$ The recently developed emulsion polymerization method to generate such small particle analogues of conjugated polymer catalysts, which can be dispersed as stable colloidal suspensions, is important in this context. ${ }^{164}$ As compared to the bulk synthesized counterparts, these emulsion polymers had nearly 2-3 times higher photocatalytic hydrogen evolution rate (Table 2) in reaction conditions where some increase in particle size due to aggregation was observed. For reaction conditions where stable dispersions were obtained without any aggregation, interestingly, the hydrogen evolution activity was lower than for the bulk polymer. This suggested that some degree of aggregation is beneficial for optimal photocatalytic activity. While the effect could in part be related to light absorption and scattering, it is also important to note that aggregation in conjugated polymers has been shown to enhance charge transport. ${ }^{165}$ In high molecular weight polymers, long and semi-rigid polymer chains lead to interconnectivity between ordered domains. Charge conduction is thus possible between the domains which lead to higher charge carrier mobility in the material. ${ }^{165,166}$ In small and medium molecular weight polymers on the other hand, such domain interconnectivity is poor and thus the grain boundaries act as transport barriers. Charge transport can thus be significantly affected by the chain length of the polymers. The molecular weight and the level of branching in the polymer further dictates the density of end groups in the polymer, which can act as trap states influencing exciton dissociation and charge transport properties of polymer photocatalysts (vide supra).

Heterojunction polymer photocatalytic systems, akin to bulk heterojunction organic photovoltaic devices (OPV), have recently been reported to be an effective approach for circumventing the problem with short exciton diffusion length and poor exciton separation in organic polymers. ${ }^{167,168}$ In such systems, exciton separation can take place at the interface of the donor conjugated polymer and an appropriate fullerene or non-fullerene acceptor. The photocatalytic HER activity of intermixed donor-acceptor blends of the donor polymer $\quad \operatorname{poly}([2,6$ '-4,8-di(5-ethylhexylthienyl)benzo[1,2- $b ; 3,3-b]$ dithiophene]\{3-fluoro-2[(2ethylhexyl)car-bonyl]thieno[3,4-b]thiophenediyl\}) (PTB7-Th) and the non-fullerene acceptor EH-IDTBR have been observed to be an order of magnitude higher than that of the corresponding core-shell photocatalysts (Table 2) and an HER rate of over $60 \mathrm{mmol} \mathrm{h}^{-1} \mathrm{~g}^{-1}$ has been reported for the former with optimized Pt co-catalyst loading. ${ }^{167}$ As compared to inorganic semiconductor materials, the solution processability of organic polymeric materials can provide a distinct advantage with regard to the generation of such bulk heterojunction architectures. 
$<$ Figure 7>

In organic polymers, the strongly bound excitons (vide supra) do not dissociate into free carriers in the bulk. Aided by the high permittivity of the aqueous medium which can screen charges from each other, exciton dissociation could take place at the polymer-water interface, ${ }^{31,169}$ similar to the dissociation of excitons in organic photovoltaics where charge separation takes place at donor-acceptor heterojunctions. ${ }^{30,170} \mathrm{~A}$ useful strategy towards achieving effective exciton dissociation was recently exemplified by a photocatalytic and transient absorption spectroscopic study of three linear conjugated polymers P1, P7 and P10. ${ }^{40}$ Long-lived electron polarons, the active species for photocatalytic hydrogen evolution, was only observed for the polymers P7 and P10 containing sulfone groups and the polaron yields increased with an increase in the number of sulfone groups. Using molecular dynamics simulation on model compounds, it could be shown that due to their hydrophilic nature, the sulfone containing polymers disperse better in the aqueous reaction mixture, by positioning themselves around the interface of the aqueous and the non-aqueous triethylamine domains with the sulfone groups preferentially aligned with water and with a shell of water established around these sulfone groups (Figure 7). Such solvent environment around the polymer affects the driving force for the electron transfer reactions, and makes the transfer of a hole to triethylamine (as well as the overall triethylamine oxidation) thermodynamically downhill (Figure 7), as opposed to an uphill reaction in the case of the polymer lacking such sulfone groups. The polymers thus produce $\mathrm{H}_{2}$ photocatalytically in the order P10 > P7 > P1 (Table 2). Besides increasing the dispersibility, hydrophilic sulfone groups can also enhance the swellability of porous polymeric photocatalysts, thus further contributing to an increase in the catalytically active surface area in the reaction mixture. ${ }^{163}$ This is important as it has been observed that microporosity can be beneficial for photocatalysis, provided it does not limit charge transport or other electronic properties.

For all organic photocatalysts, while photoactivity remains a complex interplay of a variety of highly convoluted and interlinked determinants, it is interesting to note that a broad rationalization can be found using a relatively small number of them. In a recent study of 99 dibenzo[b,d]thiophene sulfone co-polymers, the photocatalytic $\mathrm{H}_{2}$ evolution performance could be rationalized using four primary variables: electron affinity, which measures the driving force for proton reduction, ionization potential, which measures the driving force for the oxidation of the sacrificial electron donor, the optical gap regulating the light absorption, and the light transmission which is a measure of particle dispersibility. ${ }^{171}$ Based on the combination of the aforesaid four variables, a model was developed to predict the experimentally observed $\mathrm{H}_{2}$ evolution rates. Very interestingly, based on (just) these four variables, the model could predict $68 \%$ of the variation in rates, highlighting the emergence and the potential of machine learning and big data science as a new frontier for organic photocatalysis. ${ }^{172}$

$<$ Table 2>

As opposed to all advantages, a common problem with conjugated polymer photocatalysts is that they are mostly synthesized via Pd-catalyzed cross-coupling reactions and the molecular Pd catalyst can form $\operatorname{Pd}(0)$ nanoparticles by ligand loss and Ostwald ripening. Such metallic Pd nanoparticles or atomically dispersed Pd can stick to the polymer backbone and can be exceedingly difficult to completely remove. With regard to photocatalytic hydrogen evolution activity this has a profound effect as these $\operatorname{Pd}(0)$ clusters can act as highly active proton reduction electrocatalysts. This could potentially explain efficient photocatalytic $\mathrm{H}_{2}$ 
evolution of such polymers even in the absence of an externally added co-catalyst. ${ }^{151,152,157,173,174}$ Recently, a high dependence of photocatalytic $\mathrm{H}_{2}$ evolution activity on residual Pd concentration (Table 2), ${ }^{175,176}$ and an activity saturation for a residual Pd content as low as $200 \mathrm{ppm}$ was reported, thus explaining the apparent absence of trends in photocatalytic $\mathrm{H}_{2}$ evolution activity of such polymers vs $\mathrm{Pd}$ content in other reports. ${ }^{157,173}$ In the transient absorption spectroscopic study, a decreased accumulation of long lived electrons with increased quantities of residual $\mathrm{Pd}$ in the poly(9,9-dioctylfluorene-alt-benzothiadiazole) (F8BT) polymer was observed, directly showing that residual Pd catalyzes proton reduction. With regard to comparison of photocatalytic performance of the polymers under well-defined conditions and for a better deconvolution of the various factors, therefore, thorough purification and removal of residual $\mathrm{Pd}$ is necessary.

\section{Covalent triazine frameworks}

Covalent triazine frameworks (CTFs) bridge the carbon nitride class of materials and $\pi$-conjugated polymers, where the robust triazine motif from carbon nitrides forms an extended conjugated framework as in conjugated polymers (Figure 3c). ${ }^{177-180}$ Unlike carbon nitrides, CTFs possess inherent micro- and/or mesoporosity. However, the nature of the Lewis-acid catalyzed nitrile trimerization used in CTF synthesis and the high stability of the resulting triazine moiety implies low reversibility of the polymerization process. Consequently, high temperature synthesis methods have been conventionally used to construct such materials. Owing to this lack of (or poor) dynamic reversibility in triazine formation, CTFs made using ionothermal methods are mostly amorphous; Only a handful of semi-crystalline and crystalline examples are known. ${ }^{178,179,181}$ The high-temperature synthesis of such materials further leads to functional group intolerance and consequently limits the choice of monomers considerably. Also, partial carbonization occurs at such high temperatures which can be detrimental for photocatalytic applications as discussed below. ${ }^{178,182,183}$ Besides the conventional ionothermal synthesis method, there are recent reports of a variety of low temperature polymerization protocols for such materials. ${ }^{177}$ However, barring a few, ${ }^{184-188}$ most of such polymerizations occur irreversibly, and thus the final products are amorphous and functional group intolerance might still be an issue. ${ }^{177}$ The development of these protocols however significantly broadens the scope of possible monomers with which such nitrogen rich polymers could be constructed and should thus improve on their limited tunability that has impeded progress in the field of CTF photocatalysis until now.

$<$ Table 3>

The robust triazine moiety and the lack of weak bonds in the structure on the other hand endows these materials with high chemical stability. Together with the nitrogen rich backbone, tunable band gap and energy levels, this makes CTFs interesting candidates for photocatalytic $\mathrm{CO}_{2}$ reduction and water splitting. ${ }^{14,15,177,182,184,189-192}$ This feasibility was initially predicted by calculations and it was observed that the band gap of these semiconducting materials should decrease with decreasing nitrogen content in the structures. ${ }^{193}$ At the same time, a smaller pore size was calculated to induce a larger band gap by quantum confinement effects. With decreasing nitrogen content, the reducing power of the conduction band electrons was predicted to increase while the oxidizing power of the valence band holes predicted to decrease. The effect of stacking in the structure was also studied and it was found that the band gap should decrease with an increase in the number of layers, thus suggesting stacking to be an effective means to increase light harvesting and improve charge transport in such materials. CTF-T1 prepared from 1,4-dicyanobenzene in 
trifluoromethanesulfonic acid at room temperature has indeed been reported to produce hydrogen and oxygen photocatalytically under sacrificial conditions. ${ }^{194}$ However, when prepared ionothermally in $\mathrm{ZnCl}_{2}$ at high temperatures $\left(400{ }^{\circ} \mathrm{C}\right.$ ), photocatalytic $\mathrm{H}_{2}$ evolution activity was negligible (Table 3) due to framework degradation and the formation of amorphous carbon at such high temperatures, which subsequently caused huge absorption losses. ${ }^{182,195}$ An increase in the degree of polymerization and crystallinity without carbonization of CTF-1 has however been observed to increase both photocatalytic $\mathrm{H}_{2}$ and $\mathrm{O}_{2}$ evolution rates. ${ }^{182,184}$

As opposed to the polymeric CTFs, a high photocatalytic $\mathrm{H}_{2}$ evolution activity (Table 3 ), exceeding those of the benchmark carbon nitrides PTI and melon, has been observed with phenyltriazine oligomers (PTOs) synthesized by low temperature ionothermal method. ${ }^{195}$ The photocatalytic activity of the PTOs was found to be sensitive to the degree of polymerization and similar to carbon nitrides (vide supra), smaller oligomers were observed to be more active than the bigger ones down to a certain limit (Figure 8), thus suggesting a prominent role of the unreacted nitrile moieties in the photocatalytic process. While a better photocatalyst dispersion due to hydrogen bonding in the oligomers with large nitrile content was thought to be the primary reason, enhanced absorption in the visible range (possibly due to incipient carbonization, and also likely associated to a reduced stacking distance or higher torsional angle in the smaller oligomers), and more favorable carrier dynamics in the smaller oligomers were considered as contributing factors, leading to more efficient extraction of charge carriers at the interfaces. The nitrile moieties could also act as coordinating sites for $\mathrm{Pt}$ and thus a higher number of terminal nitrile moieties could promote charge transfer to the co-catalyst and thus favorably affect activity. Furthermore, the nitrile moieties may themselves act as active sites for proton reduction as the PTOs were found to be moderately active even without the addition of any co-catalyst.

$<$ Figure 8>

That crystallinity favours photocatalytic reaction yields with such materials can again be deduced from the recent report of the development of crystalline CTF-HUST-C1 by an in situ slow formation of aldehyde monomers through the controlled oxidation of alcohol precursors, ${ }^{185}$ and CTF-HUST-A1 by strong base assisted reaction between a benzylamine-functionalized monomer and an amidine monomer. ${ }^{186}$ Crystalline CTF-HUST-C1 $\left(5100 \mu \mathrm{mol} \mathrm{h}{ }^{-1} \mathrm{~g}^{-1}\right)$ and CTF-HUST-A1 $\left(9200 \mu \mathrm{mol} \mathrm{h}^{-1} \mathrm{~g}^{-1}\right)$ outperformed (Table 3) the semi-crystalline CTF-HUST-1 ${ }^{196}\left(1460 \mu \mathrm{mol} \mathrm{h} \mathrm{h}^{-1} \mathrm{~g}^{-1}\right)$ in photocatalytic $\mathrm{H}_{2}$ evolution activity and electrochemical impedance spectroscopy measurements hint to enhanced charge transport in the materials with higher crystallinity. This said, other factors like changes in light absorption and the thermodynamic driving force could also play a role. In an attempt towards identifying these factors, a series of CTFs with varied spacer units from phenylene to quarterphenylene was synthesized, and it was observed that the photocatalytic hydrogen evolution activity was a trade-off between the optical band gap that decreases with increasing number of spacer units, increasing the amount of light absorbed, and the driving force for sacrificial donor oxidation that decreases along the series. ${ }^{197}$

Similar to the study on conjugated polymers discussed in one of the preceding sections, a number of CTFs were recently synthesized and their photocatalytic $\mathrm{H}_{2}$ evolution activity was measured in a high throughput setup and the photocatalytic performance was again rationalized in terms of electron affinity, ionization potential, the optical gap and the particle dispersibility. ${ }^{198}$ Of these four properties, electron affinity and the particle dispersibility showed clear correlation with the measured hydrogen evolution activity. It was suggested that the highest photocatalytic activities correspond to specific combinations of properties. 
Nevertheless, it is important to note that CTFs with close to ideal values of these properties do not necessarily promote high hydrogen evolution rates, signifying that the four aforementioned variables are important, but not sufficient criteria for high catalytic activity. The complex interplay of the interdependent factors and the corresponding difficulty in the analysis of such bulk data is somewhat noticeable in the variation of the photoactivity $v s$ the optical gap and the PL lifetimes. Samples with large optical gap seem to have higher activity but this trend also reflects a strong correlation of the optical band gap with EA, the latter contributing to an increase in driving force for water reduction. Similarly, and again counterintuitively, samples with short excited state lifetimes seemingly elicit high photocatalytic activity. In spite of the challenging interpretation of these results, an obvious advantage of the high throughput approach is the successful identification of the most active materials in a huge parameter space (CTF-15, Table 3).

\section{Covalent organic frameworks}

The discovery of covalent organic frameworks (COFs) by Yaghi in 2005 can be thought of as the present pinnacle of the search for the fully tunable organic polymer platform. ${ }^{199}$ As photocatalysts, COFs are designed to mitigate the inherent limitations of the previously discussed classes of organic polymers and as such represent an important addition to the family of non-metal photocatalysts. One of the most relevant criteria for designing an efficient photocatalytic platform is 'tunability'. Tunability has two broad aspects, namely tuning of the polymer composition, and control of the structural order of the polymer. The molecular backbone of carbon nitrides is composed of either heptazine or triazine units, thus limiting their compositional tunability. Conjugated polymers are infinitely tunable in terms of composition, but lack control of their structural order, in other words, crystallinity. Following the recently emerging low temperature synthesis protocols, CTFs on the other hand are similar to conjugated polymers but likewise feature poor control over the structural order in most cases. Compositional tunability is straightforward as long as the structure is modular and composed of molecules. However, in a covalently linked platform the control of structural order - producing crystallinity - is difficult as typical reactions used for forming polymers are irreversible and produce kinetically driven products (Figure 3d). ${ }^{139,177}$ COFs are thus 2D or 3D polymers resulting from linking molecules using reversible reactions by thermodynamically controlled dynamic covalent chemistry (Figure 3e). ${ }^{200,201}$ The reversibility of bond formation is important as it imparts the ability of self-healing and repair of structural defects which leads to the emergence of long-range order and crystallinity. COFs are thus a significant step in the chronology of advances in organic synthesis of extended polymeric structures, ${ }^{202-208}$ and are important as photocatalysts, ${ }^{48,209-214}$ because of several reasons. They possess an extremely high chemical tunability of the activity determining factors in photocatalysis, for example light harvesting, charge separation and charge transport. Further, they possess highly tunable structural porosity beneficial for mass transport. The COF backbone is crystalline, rigid and conjugated which can enhance light harvesting and charge transport, also by reducing unproductive quenching events at structural defects. COFs are composed of covalent bonds and thus are stable and robust in principle (vide infra), as opposed to the labile metal ligand coordination bonds in metal organic frameworks (MOFs). ${ }^{48}$ Building on these advantages, in 2014 the first COF photocatalyst - the hydrazone-linked TFPT-COF was reported for HER (Table 4). ${ }^{215}$

$<$ Figure 9> 
While this proof-of-concept system marks the first example of sustained photocatalytic hydrogen evolution using a COF with quantum efficiencies competitive with carbon nitride photocatalysts, the bottom-up tunability of COFs had yet to be demonstrated. The $\mathrm{N}_{\mathrm{x}}$-COF series represents the first example where bulk properties that affect photocatalytic efficiency in such materials are tuned at the building block level. ${ }^{216}$ Increased substitution of the aromatic $\mathrm{C}-\mathrm{H}$ units with $\mathrm{N}$ atoms in the central aryl ring of a series of four azine-linked COFs gradually decreased the dihedral angle of the triaryl building block and, hence, increased the planarity of the individual 2D COF layers. This led to a progressively higher crystallinity and surface area with increasing $\mathrm{N}$ content and caused a 4-fold increase in photocatalytic $\mathrm{H}_{2}$ evolution rate with each isolobal substitution (Figure 9). The increasing $\mathrm{N}$ content also increased the electron poorness of the system, resulting in a progressive increase in the stability of the radical anion, the likely intermediate during HER after reductive quenching of the COF, thus suggesting more effective charge separation along the series. On the contrary, less symmetric molecules as monomers led to disorder and poorer crystallinity and surface area of the final COF structure and thus poor hydrogen production rates. ${ }^{217}$ As compared to the $\mathrm{N}_{\mathrm{x}} \mathrm{COF}$ systems where the activity is influenced by both electronic and structural factors, in the all planar ATEXPY-COFs the relative activities are dominated primarily by their electronic properties. ${ }^{218}$ The observed progressive decrease in photocatalytic $\mathrm{H}_{2}$ evolution rate with increasing $\mathrm{N}$ content can be related to the decreasing thermodynamic driving force for proton reduction. It is also interesting to note that while a radical anionic pathway via reductive quenching of the photoexcited COF is inferred for the aforementioned electron poor $\mathrm{N}_{\mathrm{x}}$-COFs, a radical cationic pathway via oxidative quenching is predicted for the A-TEXPYCOFs due to their electron rich nature. This points to the importance of other, more subtle features pertaining to the photocatalytic mechanism that need to be taken into account when designing a COF photocatalyst, besides the ones mentioned above like light harvesting, the thermodynamics of the reactions involved, or dispersibility.

\section{$<$ Table 4>}

The idea of enhancing catalyst wettability to improve photocatalytic performance, as developed on conjugated polymers (vide supra), was demonstrated very recently on the rigid and ordered COF platform. ${ }^{219}$ Sulfone groups in benzothiophene linkers (Figure 10) enhance the hydrophilicity of the corresponding COFs and possibly play a significant role towards increasing the photocatalytic $\mathrm{H}_{2}$ evolution rate. ${ }^{220}$ The rigid and planar structure of the benzothiophene moieties also enhances the crystallinity and porosity of such COFs, again contributing to higher activity. In fact, the dibenzothiophene sulfone containing FS-COF $\left(10100 \mu \mathrm{mol} \mathrm{h}{ }^{-1} \mathrm{~g}^{-1}\right)$ has a roughly 20 times higher photocatalytic $\mathrm{H}_{2}$ evolution rate than $\mathrm{N}_{3}$-COF $\left(470 \mu \mathrm{mol} \mathrm{h}^{-1} \mathrm{~g}^{-1}\right)$ when measured under similar conditions and the highest achieved to date in non-sensitized COF based systems. The ordered nature and the hydrophilicity of the mesopores further aid external sensitization of the COF using an infrared absorbing dye, thus increasing the absorption cross section of the system to longer wavelengths and consequently the $\mathrm{H}_{2}$ evolution rate increased by more than 1.5 times compared to the unsensitized system (Table 4 ).

\section{$<$ Figure 10>}

The highly tunable crystalline platform in COFs comes at a price. While the reversibility in bond formation is necessary for crystallinity of the final structure, it can also make the COF less stable and vulnerable to 
hydrolysis because bond breaking is as facile as the bond formation process. This is especially true for COFs with boron-based linkages and most COFs synthesized via Schiff-base chemistry, the latter representing the largest subgroup of COFs to date. ${ }^{207,221-223}$ While COFs with boron-based linkages are not stable in water, ${ }^{224-226}$ most imine-based COFs have limited stability in water under acidic or alkaline conditions. ${ }^{23,227-229}$ Consequently, in terms of a stable platform for photocatalysis, the COF linkage could very well be the weakest link. One possibility to alleviate this problem is to use $\beta$-ketoenamine, ${ }^{230,231}$ or thiazole-linked COFs, ${ }^{232}$ which are "locked" versions of the initially formed imine COFs. Likewise, the newly developed olefin-linked COFs were recently demonstrated as another means of circumventing this problem (Figure 10). ${ }^{233-237}$ In one such COF platform, a monofunctionalized linker was used to cap the free ends of the electron rich pyrene-based COF with an electron accepting 3-ethylrhodanine unit, thus creating an integrated donor-acceptor heterojunction in the structure. ${ }^{238}$ This enhanced the light absorption, charge separation and charge transport properties of the $\mathrm{COF}$, and the photocatalytic $\mathrm{H}_{2}$ evolution rate increased (to $2120 \quad \mu \mathrm{mol} \quad \mathrm{h}^{-1} \quad \mathrm{~g}^{-1}$ ) compared to the uncapped COF $\left(1360 \mu \mathrm{mol} \mathrm{h}^{-1} \mathrm{~g}^{-1}\right)$. The absence of iminic nitrogens that could deactivate the excited state and the complete conjugation in the structure (compared to the limited conjugation in meta-linked systems like the $\mathrm{N}_{\mathrm{x}}$-COFs) were proposed as additional factors contributing to improved activity (Table 4).

Due to the difficulties associated with solution processing and thin film fabrication, photophysical studies to elucidate the excited state dynamics of COFs are still rare. ${ }^{41,239,240}$ In one of the first such studies, the efficacy of donor-acceptor COF structures in facilitating charge separation and suppressing recombination was demonstrated in a boronate ester linked Zinc phthalocyanine ( $\mathrm{ZnPc}$ ) donor - naphthalene diimide (NDI) acceptor COF. ${ }^{239}$ Transient absorption spectroscopy of the benzonitrile COF suspension revealed ultrafast electron transfer within $1.4 \mathrm{ps}$ from ${ }^{1} \mathrm{ZnPc} *$ to $\mathrm{NDI}$, in the self-sorted, columnar donor and acceptor heterojunction structure. This was followed by charge delocalization in the $\pi$-columns and the lifetime of the hence formed charge separated state was measured to be as long as $\sim 10 \mu \mathrm{s}$ in benzonitrile, as compared to $217 \mathrm{ps}$ in dimethylformamide (DMF) where the COF is delaminated into few layer stacks. This suggested that the layered COF structure is crucial for prolonged charge separation. Recently the excited state dynamics of vertically oriented thin films of fully conjugated imine linked COFs composed of tetraphenylethylene nodes and thienothiophene linkers was studied. ${ }^{240}$ The singlet excitons created upon photoexcitation were suggested to diffuse through the conjugated COF network, both laterally and across the sheets, and ultimately undergo singlet-singlet annihilation (SSA) to create free long-lived charges with lifetimes of several tens of microseconds. A structural and mechanistic understanding of the exact role of the COF structure in "trapping" and prolonging the lifetime of the free charges would however be expedient to translate these insights into the rational design of improved COF photocatalysts.

The ordered structure of the COF backbone and the pores, and the ability to control the order at the atomic or molecular level opens up the prospect of achieving atomically distributed, isolated, uniform, and spatially separated active centers on such platforms, that is, the development of single-site photocatalysts. ${ }^{48,241}$ This can reduce the usage of rare and expensive noble metal co-catalysts, can provide well-defined systems to understand the intrinsic catalytic mechanism (and thereby tune it), and ultimately could lead to the development of a 'COF leaf' - which integrates and orchestrates different electronically coupled subunits on a single platform to drive and facilitate light-induced water splitting and $\mathrm{CO}_{2}$ reduction. It is important to note that molecular co-catalysts have been coupled before with organic polymers, ${ }^{242}$ especially with carbon nitrides, for photocatalytic $\mathrm{H}_{2},{ }^{243,244} \mathrm{O}_{2}$ evolution, ${ }^{245}$ and $\mathrm{CO}_{2}$ reduction ${ }^{246,247}$. However, the typically 
poor structural control renders understanding and manipulation of the systems more difficult than in COFs. ${ }^{248,249}$

$<$ Figure 11>

The development of heterogeneous single-site systems is challenging because it implies the use of molecular single-site catalysts, which quite generally have limited photostability. For a single-site heterogeneous photocatalytic COF platform, generally slow multielectron, diffusion-controlled catalytic processes need to be coupled efficiently to the light-harvesting and charge-percolating COF. This was first demonstrated using cobaloxime proton reduction co-catalysts with the $\mathrm{N}_{\mathrm{x}}$-COFs and hydrazone-linked COF-42 photosensitizers (Table 4). ${ }^{250} \mathrm{H}_{2}$ evolution activity was found to be dependent on the solvent, reaction $\mathrm{pH}$, choice of sacrificial donor, and the crystallinity and porosity of the COF. Further, the singlesite nature of the photocatalytic system enabled spectroscopic identification of reaction intermediates in the proton reduction process for the first time. Interestingly, with metallic Pt co-catalyst, a three times lower $\mathrm{H}_{2}$ evolution rate was observed compared to that with cobaloxime co-catalyst, most likely due to a poorer distribution and/or photodeposition of $\mathrm{Pt}$ nanoparticles on the COF surface. Photodeposition and distribution of metallic nanoparticles on the photocatalyst surface, and the sizes of the nanoparticle clusters, only the surface atoms of which are catalytically active, thus are factors that affect photoactivity. More recently, sustained photocatalytic $\mathrm{H}_{2}$ evolution using such single-site systems under aqueous conditions was reported using a thiazolothiazole-bridged TpDTz COF and a nickel-thiolate cluster co-catalyst in the presence of triethanolamine as the sacrificial electron donor. ${ }^{251}$ Microkinetic modeling of the reaction suggested the outer-sphere electron transfer from the COF to the co-catalyst to be the rate-limiting step, highlighting the importance of a rational design of the $\mathrm{COF}-$ co-catalyst interface. In this regard, improving upon the weak and nonspecific interaction between the COF and the co-catalyst by covalently linking the two could be beneficial and would lead to a true single-site COF-based photocatalytic system. This was recently achieved using azide-functionalized chloro(pyridine)cobaloxime HER co-catalysts covalently linked to hydrazone-based COF-42. ${ }^{252}$ This chemisorbed COF-co-catalyst hybrid was found to be photocatalytically more active than the corresponding physisorbed mixture at the same co-catalyst loading level. Advanced solid-state nuclear magnetic resonance (NMR) and quantum chemical calculations revealed interaction of the chemisorbed co-catalyst with the COF backbone. Such interaction possibly facilitates re-attachment of the co-catalyst to the COF during the photoreaction, thereby preventing its degradation and increasing the longevity of the photocatalytic system. The COF- $\operatorname{Re}(\mathrm{CO})_{3} \mathrm{Cl}$ covalently linked system for photocatalytic $\mathrm{CO}_{2}$ reduction to $\mathrm{CO}$ is another significant example of true single-site COF-based photocatalytic system (Table 4). ${ }^{249}$ Using transient absorption spectroscopy and ground state spectroscopic measurements, a detailed mechanism for the photocatalytic $\mathrm{CO}_{2}$ reduction process could be elucidated (Figure 11). It was observed that an intramolecular charge transfer state initially forms upon photoexcitation where electrons are partially located in the $\mathrm{Re}(\mathrm{bpy})(\mathrm{CO})_{3} \mathrm{Cl}$ unit. This enhances the lifetime of the intramolecular charge transfer state, thereby kinetically favoring the formation of the subsequent $\mathrm{TEOA}^{+}-(\mathrm{COF})^{-}$charge separated state. This charge separated state was then proposed to activate $\mathrm{CO}_{2}$ via coordination to the Re center and concomitant with the dissociation of $\mathrm{Cl}^{-}$. 


\section{Outlook}

Due to differences in the nature of excitons as well as the availability and mobility of free charge carriers, there exist significant differences between inorganic and organic semiconductor photocatalysts in terms of charge separation and transport, which necessitates a fresh look at the photophysical and physicochemical principles governing photocatalysis, referenced to the specific properties of organic materials. Beyond doubt, polymer photocatalysts present an enormous scope in terms of structure - property relationships due to their unique chemical versatility, allowing targeted tuning of building blocks to modulate their photophysical and physicochemical properties. Although our present understanding of a specific structural change and its implications is often based on correlations, more and more fundamental studies can and will lead to a rational property design in such materials.

From the examples discussed in this article, one of the most obvious corollaries is that for such organic polymeric photocatalysts, activities are a complex interplay of an array of factors - factors that can be highly interdependent. Furthermore, the interdependence can be a function of the specific type of organic polymer platform, complicating the understanding even further. There are however some readily identifiable determinants that influence the photocatalytic efficiency of organic polymers. As in inorganic semiconductor systems these include electronic properties such as light harvesting, exciton dissociation, charge transport and thermodynamic driving force for the surface catalytic reactions on the one hand, and structural properties like crystallinity, surface area, particle size and dispersibility or catalyst wettability on the other. The majority of studies on different polymer classes suggest that crystallinity of the organic polymer is one of the most important determinants, affecting charge carrier lifetime and mobility, thereby influencing photocatalytic efficiency. Crystallinity can further affect photodeposition of metallic cocatalysts, ${ }^{219}$ and can be advantageous for the development of single-site heterogeneous photocatalytic systems.

As current studies project, a grand challenge in polymer photocatalysis is the understanding of the nature and role of defects and disorder, which can have multiple intertwined functions affecting light harvesting, charge transport, and catalysis in often opposing and counter-intuitive directions. Thus, more effort needs to be expended in order to identify, incorporate and discern the exact role of defects and disorder in the respective polymer structures, making surface engineering of polymer photocatalysts important as we move towards a self-standing, earth-abundant artificial photosynthetic platform.

In the absence of polymers with built-in active sites, it is a common approach to separate light absorption from chemical catalysis with the use of appropriate co-catalysts for proton reduction, water oxidation and $\mathrm{CO}_{2}$ reduction. In such polymer-co-catalyst conjugates, the photoabsorber - co-catalyst interface, especially the charge transfer mechanism from the photoabsorber to the co-catalyst, needs to be better understood and rationally engineered for optimal photocatalytic performance, ideally within a fully earth-abundant photocatalytic system. This said, a future frontier in "soft photocatalysis" will be the development of polymers with intrinsic catalytic sites that chemically and spatially belong to the polymer backbone, or are atomically dispersed on it in the form of single metal atoms that are structurally bound to the polymer by strong metal-support interactions. ${ }^{253}$

What is still predominantly lacking in the field of photocatalysis using organic polymeric materials is a thorough molecular level mechanistic understanding of the fundamental processes in the respective polymeric platform. This mandates that investigations must be done systematically and more fundamentally such that photocatalysts are first astutely designed and then studied comprehensively to understand the complete picture. In the same vein, but on a more practical note, reporting and comparison of photocatalytic 
performance parameters should be revised to guarantee more objective measures of photocatalytic activity, which currently are non-standardized and thus ambiguous. This includes the establishment of best-practice approaches to measurement, data analysis and reactor design, but also the reporting of standardized performance parameters, which widely differs between laboratories, making general statements or comparisons of photocatalytic activities unreliable, if not impossible.

What comes next? The fact that most phenomenological investigations based on the convoluted set of interdependent parameters often do not translate into clear-cut variations in photoactivity suggests that future research must take on innovative new directions. These should primarily be geared towards deconvoluting the photocatalytic system to optimize each step in the photocatalytic cycle individually, and to introduce new concepts that take advantage of the unique tunability of polymer photocatalysts. This can include the use of dedicated photosensitizers, ${ }^{219,254}$ and introduction of concepts like molecular upconversion, ${ }^{255}$ and singlet fission, ${ }^{256}$ to enhance photoabsorption of such molecularly tunable heterogeneous platforms.

Engineering the porosity of the polymer photocatalysts to study effects of confinement of substrates during photocatalysis and the role of hierarchical porosity ${ }^{257}$ in facilitating mass transport during photocatalysis likewise present interesting research avenues. Another exciting photocatalytic platform would be polymers with built-in functional groups such as the recently reported sub-stoichiometric COFs where the free functional moieties (which could act as potential defects or catalyst binding sites) can be controlled and positioned with atomic level precision. ${ }^{258} 3 \mathrm{D}$ architectures, which have received little attention in photocatalysis so far, can serve as topologically interesting photocatalytic platforms as well, ${ }^{259}$ as they may facilitate isotropic charge transport and the design of bulk heterojunction architectures, if astutely designed. In $3 \mathrm{D}-\mathrm{COFs},{ }^{260}$ with pore size engineering, the possibility of substrate and/or co-catalyst confinement might actually outweigh the apparent hindrances associated with the lack of a conjugated structure which can deleteriously affect light absorption and charge percolation during photocatalysis as compared to 2D COFs.

Another important future research direction will be the quest for photocatalytic platforms that avoid the use of sacrificial components which do not lead to value-added products. Where overall water splitting is the ultimate goal, realizing stable and efficient organic polymer photocatalyst systems with a high-enough driving force for water oxidation and minimal recombination loss remains an ambitious task. ${ }^{261,262} \mathrm{~A}$ probable solution could be the use of two photocatalysts forming a charge separating junction such that the electrons and holes are spatially separated on sub-systems minimizing kinetic limitations associated with the four-electron water oxidation process. ${ }^{263} \mathrm{With}$ regard to overall water splitting, it is also important to note that oxygen accumulated from the photoreaction can act as an electron scavenger, and thus directly reduce the yield of the reduction product while forming unwanted reactive oxygen species. The accumulation of oxyhydrogen gas can further be dangerous and will require an additional separation step, at the expense of the energy stored in the solar fuels. Thus, for maintaining practical photocatalytic activities in the long run, the reduction and oxidation reactions should ideally be separated into different 'reaction chambers', putting photoelectrochemical schemes into the focus. In terms of devising complex multicomponent architectures where individual half reactions of the artificial photosynthetic processes are orchestrated to act in tandem, and also for the fabrication of heterojunction architectures for efficient excitonic separation, the solution processability and thin film fabrication with organic polymers is crucial. Film fabrication should also facilitate in situ or operando spectroscopic studies of the fundamental photophysics of the polymer and the photocatalytic process in detail. 
Finally, one needs to address the issue with the long-term stability of organic polymer photocatalysts, especially regarding COFs with labile bonds. While the tunability of such platforms adds a potential advantage in this regard (vide supra), one needs to look not only at the intrinsic long-term stability but also explore possible catalyst poisoning mechanisms in sufficient detail.

Looking at the rapidly evolving field of polymer photocatalysis, one may be tempted to ask: which is the best organic polymeric photocatalyst? At this stage, this question is rather ill-posed as the amount of data is still too small to pinpoint which features are most effective in determining the photocatalytic activity and how they interact on each polymer platform. While the answer also depends on the specific reaction and a case-to-case dependent trade-off between cost, scalability, performance, long term stability and recyclability, the inherent shortcoming puts the spotlight on the powerful combination of experimental high-throughput screening approaches and computational design by machine learning, which has recently entered the scene of "soft photocatalysis" and is expected to drive this field in the years to come. ${ }^{171,172,264,265}$ But already now, it is clear from the above that the high level of inherent tunability of organic polymeric photocatalysts sets them apart from their inorganic semiconductor analogues so that many impediments in charge separation and transport can be rationally addressed, in principle. A key ingredient of future progress will therefore be to map out the huge structure-property-activity space in these materials by a clever combination of chemical and computational design. The present-day success and level of insight is only the tip of the iceberg. In future, organic polymers will further mature and likely secure a firm place among the most promising classes of photocatalysts as we learn to understand and improve the bottom-up design of these materials for photocatalytic applications. 
Table 1. Summary of photocatalytic $\mathrm{H}_{2}$ evolution activity of selected carbon nitrides.

\begin{tabular}{|c|c|c|c|c|c|}
\hline Material & Reaction conditions & $\begin{array}{c}\text { Activity } \\
\left(\mu \mathrm{mol} \mathrm{h}{ }^{-1} \mathrm{~g}^{-1}\right)\end{array}$ & AQE & Remarks & Reference \\
\hline \multirow[t]{4}{*}{ Melon } & $\begin{array}{c}10 \text { vol } \% \text { TEOA in } \\
\text { water, }>420 \mathrm{~nm}\end{array}$ & $1-40^{a}$ & & First report & 71 \\
\hline & $\begin{array}{l}10 \text { vol } \% \text { TEOA in } \\
\text { water, } \mathrm{Pt},>420 \mathrm{~nm}\end{array}$ & 722 & $\begin{array}{l}0.5 \% \text { at } \\
400 \mathrm{~nm}\end{array}$ & & 75 \\
\hline & $\begin{array}{c}10 \mathrm{vol} \% \mathrm{MeOH} \text { in } \\
\text { water, Pt, AM } 1.5 \mathrm{G}\end{array}$ & 25 & & & 76 \\
\hline & $\begin{array}{l}10 \mathrm{vol} \% \text { TEOA in } \\
\text { water, } \mathrm{Pt},>420 \mathrm{~nm}\end{array}$ & $5000^{a}$ & $\begin{array}{l}6.3 \% \text { at } \\
420 \mathrm{~nm}\end{array}$ & $\begin{array}{l}4 \mathrm{~nm} \text { thick } \\
\text { nanosheets }\end{array}$ & 119 \\
\hline $\mathrm{PTI} / \mathrm{Li}^{+} \mathrm{Cl}^{-}$ & $\begin{array}{c}10 \mathrm{vol} \% \text { TEOA in } \\
\text { water, } \mathrm{Pt},>420 \mathrm{~nm}\end{array}$ & 864 & $\begin{array}{l}0.6 \% \text { at } \\
400 \mathrm{~nm}\end{array}$ & & 75 \\
\hline K-PHI & $\begin{array}{c}10 \mathrm{vol} \% \mathrm{MeOH} \text { in } \\
\text { water, Pt, AM } 1.5 \mathrm{G}\end{array}$ & 600 & & $\begin{array}{l}\text { Small crystallite sizes } \\
\qquad(\sim 20 \mathrm{~nm})\end{array}$ & 76 \\
\hline H-PHI & $\begin{array}{l}10 \text { vol } \% \mathrm{MeOH} \text { in } \\
\text { water, Pt, AM } 1.5 \mathrm{G}\end{array}$ & 3364 & & $\begin{array}{l}\text { Small crystallite sizes } \\
\qquad(\sim 20 \mathrm{~nm})\end{array}$ & 76 \\
\hline $\mathrm{NS}-\mathrm{g}-\mathrm{C}_{3} \mathrm{~N}_{4}$ & $\begin{array}{l}10 \mathrm{vol} \% \text { TEOA in } \\
\text { water, } \mathrm{Pt},>420 \mathrm{~nm}\end{array}$ & $14350^{a}$ & $\begin{array}{l}9.6 \% \text { at } \\
420 \mathrm{~nm}\end{array}$ & $\begin{array}{c}\text { Silica spheres as } \\
\text { sacrificial templates } \\
\text { to get nanospherical } \\
\text { structure composed } \\
\text { of nanosheets, } \\
\text { surface area of } 160 \\
\mathrm{~m}^{2} \mathrm{~g}^{-1}\end{array}$ & 112 \\
\hline $\begin{array}{l}\text { Oligomers of } \\
\text { melem }\end{array}$ & $\begin{array}{c}1 \mathrm{vol} \% \mathrm{MeOH} \text { in } 0.1 \\
\mathrm{M} \text { PBS buffer at } \mathrm{pH} \\
7, \mathrm{Pt},>420 \mathrm{~nm}\end{array}$ & $270^{a}$ & $\begin{array}{l}0.1 \% \text { at } \\
400 \mathrm{~nm}\end{array}$ & $\begin{array}{c}0.9 \\
\mu \mathrm{mol} \mathrm{h} \mathrm{h}^{-1} \text { for } \\
\text { polymeric melon } \\
\text { under similar } \\
\text { conditions }\end{array}$ & 122 \\
\hline $\begin{array}{l}\text { Infusion of } \\
\text { bulk and } \\
\text { exfoliated } \\
\text { melon }\end{array}$ & $\begin{array}{l}10 \text { vol \% TEOA in } \\
\text { water, } \mathrm{Pt}, 365 \mathrm{~nm}\end{array}$ & 3810 & & $\begin{array}{c}\text { Importance of } \\
\text { terminal amine } \\
\text { groups in exfoliated } \\
\text { melon }\end{array}$ & 123 \\
\hline
\end{tabular}


Table 2. Summary of photocatalytic $\mathrm{H}_{2}$ evolution activity of selected conjugated polymers. ${ }^{a}$

\begin{tabular}{|c|c|c|c|c|c|}
\hline Material & Reaction conditions & $\begin{array}{c}\text { Activity } \\
\left(\mu \mathrm{mol} \mathrm{h}{ }^{-1} \mathrm{~g}^{-1}\right)\end{array}$ & AQE & Remarks & Reference \\
\hline $\begin{array}{l}\text { Poly-p- } \\
\text { phenylene }\end{array}$ & $\begin{array}{l}\text { 1:1 DEA, water, } \\
>290 \mathrm{~nm}\end{array}$ & $105^{b}$ & $<0.04 \%$ & First report & 136 \\
\hline CP-CMP10 & $\begin{array}{l}20 \text { vol } \% \text { DEA in } \\
\text { water, }>420 \mathrm{~nm}\end{array}$ & $174^{b}$ & & & 157 \\
\hline P10e & $\begin{array}{c}1: 1: 1 \\
\text { water:TEA:MeOH } \\
\text { (aqueous phase } \\
\text { containing } \\
\text { water : toluene } 9: 1, \\
\text { SDS surfactant } 10 \mathrm{mg} \\
\mathrm{mL}^{-1} \text { and } \mathrm{Na}_{2} \mathrm{CO}_{3} 3.5 \\
\mathrm{mg} \mathrm{mL}^{-1} \text { ), }>420 \mathrm{~nm}\end{array}$ & 14520 & & $\begin{array}{c}\text { Emulsion } \\
\text { polymerized particle }\end{array}$ & 164 \\
\hline \multirow[t]{2}{*}{$\mathrm{P} 10$} & Same as P10e & 6130 & & & 164 \\
\hline & $\begin{array}{c}\text { 1:1:1 } \\
\text { water:TEA:MeOH, } \\
>420 \mathrm{~nm}\end{array}$ & 3260 & $\begin{array}{l}11.6 \% \text { at } \\
420 \mathrm{~nm}\end{array}$ & $\begin{array}{l}\text { Hydrophilicity of the } \\
\text { polymer backbone }\end{array}$ & 40 \\
\hline P7 & $\begin{array}{c}\text { 1:1:1 } \\
\text { water:TEA:MeOH, } \\
>420 \mathrm{~nm}\end{array}$ & $1492^{b}$ & $\begin{array}{l}7.2 \% \text { at } \\
420 \mathrm{~nm}\end{array}$ & & 40 \\
\hline $\mathrm{P} 1$ & $\begin{array}{c}\text { 1:1:1 } \\
\text { water:TEA:MeOH, } \\
>420 \mathrm{~nm}\end{array}$ & $64^{b}$ & $\begin{array}{l}0.4 \% \text { at } \\
420 \mathrm{~nm}\end{array}$ & & 40 \\
\hline S-CMP3 & $\begin{array}{c}\text { 1:1:1 } \\
\text { water:TEA:MeOH, } \\
>420 \mathrm{~nm}\end{array}$ & 3106 & $\begin{array}{l}13.2 \% \text { at } \\
420 \mathrm{~nm}\end{array}$ & $\begin{array}{l}\text { Microporous network } \\
\text { (compare to the non- } \\
\text { porous linear polymer } \\
\text { P-35 below) }\end{array}$ & 163 \\
\hline $\mathrm{P}-35$ & $\begin{array}{c}\text { 1:1:1 } \\
\text { water:TEA:MeOH, } \\
>420 \mathrm{~nm}\end{array}$ & 826.1 & & & 163 \\
\hline \multirow[t]{2}{*}{$\begin{array}{c}\text { F8BT } \\
\text { nanoparticles }\end{array}$} & $\begin{array}{c}\text { 3:7 DEA:water, } \\
\text { 385-700 nm }\end{array}$ & 176.6 & $\begin{array}{l}0.02 \% \text { at } \\
340 \mathrm{~nm}\end{array}$ & 1170 ppm residual Pd & 175 \\
\hline & $\begin{array}{l}\text { 3:7 DEA:water; } \\
\text { 385-700 nm }\end{array}$ & $\mathrm{No} \mathrm{H}_{2}$ & & $<1 \mathrm{ppm}$ residual $\mathrm{Pd}$ & 175 \\
\hline $\begin{array}{l}\text { PTB7- } \\
\text { Th:EH- }\end{array}$ & $\begin{array}{c}0.2 \mathrm{~mol} \mathrm{~L}^{-1} \text { ascorbic } \\
\text { acid, } \mathrm{pH} 2, \mathrm{Pt}, 350- \\
800 \mathrm{~nm}\end{array}$ & $3044 \pm 332$ & & $\begin{array}{l}\text { Core-shell donor- } \\
\text { acceptor nanoparticle }\end{array}$ & 167 \\
\hline
\end{tabular}




\section{IDTBR}

SDS, 10:90

$\begin{array}{cccc}\text { PTB7- } & 2 \mathrm{~mol} \mathrm{~L}^{-1} \text { ascorbic } & 28,133 \pm & \text { Intermixed } \\ \text { Th:EH- } & \text { acid, } \mathrm{pH} \mathrm{2,} \mathrm{Pt,350-} & 3,067 & \text { nanoparticle } \\ \text { IDTBR } & 800 \mathrm{~nm} & & \text { morphology }\end{array}$

TEBS, 30:70

\footnotetext{
${ }^{a}$ Note the presence of residual Pd for all entries except the first, unless otherwise mentioned.

${ }^{b}$ Re-calculated from the reported values.
}

AQE - Apparent Quantum Efficiency, DEA - Diethylamine, SDS - Sodium dodecyl sulfate. TEA - Triethylamine. TEBS - sodium 2-(3-thienyl)ethyloxybutylsulfonate. 
Table 3. Summary of photocatalytic $\mathrm{H}_{2}$ evolution activity of selected CTF-based systems.

\begin{tabular}{|c|c|c|c|c|c|}
\hline Material & Reaction conditions & $\begin{array}{c}\text { Activity } \\
\left(\mu \mathrm{mol} \mathrm{h}{ }^{-1} \mathrm{~g}^{-1}\right)\end{array}$ & AQE & Remarks & Reference \\
\hline CTF-1 & $\begin{array}{c}10 \text { vol } \% \text { TEOA, } 0.5 \\
\text { M phosphate buffer at } \\
\text { pH } 7, \mathrm{Pt}, \mathrm{AM} 1.5 \mathrm{G}\end{array}$ & 0.02 & & $\begin{array}{l}\text { CTF synthesized } \\
\text { ionothermally at } \\
\text { high temperature, } \\
\text { carbonization }\end{array}$ & 195 \\
\hline CTF-1 & $\begin{array}{c}3 \text { vol } \% \mathrm{MeOH} \text { and } 10 \\
\text { vol \% TEOA in water, } \\
\mathrm{Pt},>420 \mathrm{~nm}\end{array}$ & 5500 & $\begin{array}{l}6 \% \text { at } \\
420 \\
\mathrm{~nm}\end{array}$ & $\begin{array}{l}\text { CTF synthesized } \\
\text { by mild } \\
\text { microwave- } \\
\text { assisted } \\
\text { polymerization }\end{array}$ & 184 \\
\hline PTO-300 & $\begin{array}{c}10 \text { vol \% TEOA, } 0.5 \\
\text { M phosphate buffer at } \\
\text { pH } 7, \mathrm{Pt}, \mathrm{AM} 1.5 \mathrm{G}\end{array}$ & 1076 & $\begin{array}{l}5.5 \% \\
\text { at } 400 \\
\mathrm{~nm}\end{array}$ & $\begin{array}{l}\text { Oligomers as } \\
\text { photocatalysts }\end{array}$ & 195 \\
\hline $\begin{array}{c}\text { CTF-HUST- } \\
\text { C1 }\end{array}$ & $\begin{array}{c}10 \text { vol } \% \text { TEOA in } \\
\text { water } \\
, \mathrm{Pt},>420 \mathrm{~nm}\end{array}$ & 5100 & & $\begin{array}{l}\text { Crystalline CTF } \\
\text { synthesized by in } \\
\text { situ formation of } \\
\text { aldehyde } \\
\text { monomers }\end{array}$ & 185 \\
\hline $\begin{array}{c}\text { CTF-HUST- } \\
\text { A1 }\end{array}$ & $\begin{array}{c}10 \text { vol } \% \text { TEOA in } \\
\text { water } \\
, \mathrm{Pt},>420 \mathrm{~nm}\end{array}$ & 9200 & $\begin{array}{c}7.4 \% \\
\text { at } 420 \\
\mathrm{~nm}\end{array}$ & $\begin{array}{l}\text { Crystalline CTF } \\
\text { synthesized by } \\
\text { strong base } \\
\text { assisted reaction } \\
\text { between amidine } \\
\text { and benzylamine- } \\
\text { functionalized } \\
\text { monomers }\end{array}$ & 186 \\
\hline CTF-15 & $\begin{array}{c}1.1: 1 \\
\text { water:TEA:MeOH, } \\
\mathrm{Pt},>420 \mathrm{~nm}\end{array}$ & 1909 & $\begin{array}{c}15.9 \% \\
\text { at } 420 \\
\text { nm }\end{array}$ & $\begin{array}{l}\text { High throughput } \\
\text { workflow }\end{array}$ & 198 \\
\hline
\end{tabular}

AQE - Apparent Quantum Efficiency, CTF - Covalent Triazine Framework, TEA - Triethylamine, TEOA Triethanolamine. 
Table 4. Summary of photocatalytic activity of selected COF-based systems.

\begin{tabular}{|c|c|c|c|c|c|c|c|}
\hline Material & $\begin{array}{c}\text { Photocatalytic } \\
\text { reaction }\end{array}$ & $\begin{array}{c}\text { Reaction } \\
\text { conditions }\end{array}$ & Activity & $\overline{A Q E}$ & TON & Remarks & Reference \\
\hline $\begin{array}{l}\text { TFPT- } \\
\text { COF }\end{array}$ & $\mathrm{H}_{2}$ evolution & $\begin{array}{c}1 \mathrm{wt} \% \\
\text { sodium } \\
\text { ascorbate in } \\
\text { water, } \mathrm{Pt} \text {, } \\
>420 \mathrm{~nm}\end{array}$ & $\begin{array}{c}230 \\
\mu \mathrm{mol} \\
\mathrm{h}^{-1} \mathrm{~g}^{-1}\end{array}$ & & & First report & 215 \\
\hline $\mathrm{N}_{3}-\mathrm{COF}$ & $\mathrm{H}_{2}$ evolution & $\begin{array}{c}1 \mathrm{vol} \% \\
\text { TEOA, } \\
\text { Phosphate } \\
\text { buffer at } \\
\mathrm{pH} \mathrm{7,} \mathrm{Pt,} \\
>420 \mathrm{~nm}\end{array}$ & $\begin{array}{c}1703 \\
\mu \mathrm{mol} \\
\mathrm{h}^{-1} \mathrm{~g}^{-1}\end{array}$ & $\begin{array}{c}0.44 \% \\
\text { at } 450 \\
\mathrm{~nm}\end{array}$ & & & 216 \\
\hline $\mathrm{FS}-\mathrm{COF}$ & $\mathrm{H}_{2}$ evolution & $\begin{array}{c}0.1 \mathrm{M} \\
\text { ascorbic acid } \\
\text { in water, } \mathrm{Pt} \\
>420 \mathrm{~nm}\end{array}$ & $\begin{array}{c}10100 \\
\mu \mathrm{mol} \\
\mathrm{h}^{-1} \mathrm{~g}^{-1}\end{array}$ & $\begin{array}{c}3.2 \% \\
\text { at } 420 \\
\mathrm{~nm}\end{array}$ & & $\begin{array}{l}\text { Sulfone } \\
\text { groups for } \\
\text { increased } \\
\text { wettability }\end{array}$ & 219 \\
\hline $\begin{array}{c}\text { FS- } \\
\text { COF+WS5F } \\
\text { dye }\end{array}$ & $\mathrm{H}_{2}$ evolution & $\begin{array}{c}0.1 \mathrm{M} \\
\text { ascorbic acid } \\
\text { in water, } \mathrm{Pt} \\
>420 \mathrm{~nm}\end{array}$ & $\begin{array}{c}16300 \\
\mu \mathrm{mol} \\
\mathrm{h}^{-1} \mathrm{~g}^{-1}\end{array}$ & $\begin{array}{c}2.2 \% \\
\text { at } 600 \\
\mathrm{~nm}\end{array}$ & & $\begin{array}{c}\text { Dye } \\
\text { sensitization }\end{array}$ & 219 \\
\hline $\begin{array}{c}\mathrm{sp}^{2} \mathrm{c}- \\
\mathrm{COF} \\
\text { ERDN }\end{array}$ & $\mathrm{H}_{2}$ evolution & $\begin{array}{l}10 \mathrm{vol} \% \\
\text { TEOA in } \\
\text { water, } \mathrm{Pt} \text {, } \\
>420 \mathrm{~nm}\end{array}$ & $\begin{array}{c}2120 \\
\mu \mathrm{mol} \\
\mathrm{h}^{-1} \mathrm{~g}^{-1}\end{array}$ & $\begin{array}{c}0.48 \% \\
\text { at } 495 \\
\mathrm{~nm}\end{array}$ & & $\begin{array}{l}\text { Olefin-linked } \\
\text { COFs with } \\
\text { donor-acceptor } \\
\text { structure }\end{array}$ & 238 \\
\hline $\mathrm{N}_{2}-\mathrm{COF}$ & $\mathrm{H}_{2}$ evolution & $\begin{array}{c}1 \text { vol } \% \\
\text { TEOA, } 4: 1 \\
\text { ACN: } \mathrm{H}_{2} \mathrm{O} \text { at } \\
\mathrm{pH} 8,0.1 \mathrm{mM} \\
\text { cobaloxime, } \\
60 \text { equiv } \\
\text { dmgH }_{2}, \\
\text { AM } 1.5 \mathrm{G}\end{array}$ & $\begin{array}{c}782 \\
\mu \mathrm{mol} \\
\mathrm{h}^{-1} \mathrm{~g}^{-1}\end{array}$ & $\begin{array}{c}0.16 \% \\
\text { at } 400 \\
\mathrm{~nm}\end{array}$ & 54.4 & $\begin{array}{l}\text { Physisorbed } \\
\text { molecular } \\
\text { proton } \\
\text { reduction co- } \\
\text { catalyst }\end{array}$ & 250 \\
\hline $\mathrm{Re}-\mathrm{COF}$ & $\begin{array}{c}\mathrm{CO}_{2} \\
\text { photoreduction }\end{array}$ & $\begin{array}{l}5 \mathrm{vol} \% \\
\text { TEOA in } \\
\text { ACN, } \\
>420 \mathrm{~nm}\end{array}$ & $\begin{array}{c}15 \\
\mathrm{mmol} \\
\mathrm{CO}_{2} / \mathrm{g} \\
\text { of } \mathrm{COF} \\
\text { in } \\
>20 \mathrm{~h}\end{array}$ & - & 48 & $\begin{array}{c}\text { True single- } \\
\text { site } \mathrm{CO}_{2} \\
\text { photoreduction }\end{array}$ & 249 \\
\hline
\end{tabular}

ACN - Acetonitrile, COF - Covalent Organic Framework, TEOA - Triethanolamine, TON - Turnover number. 


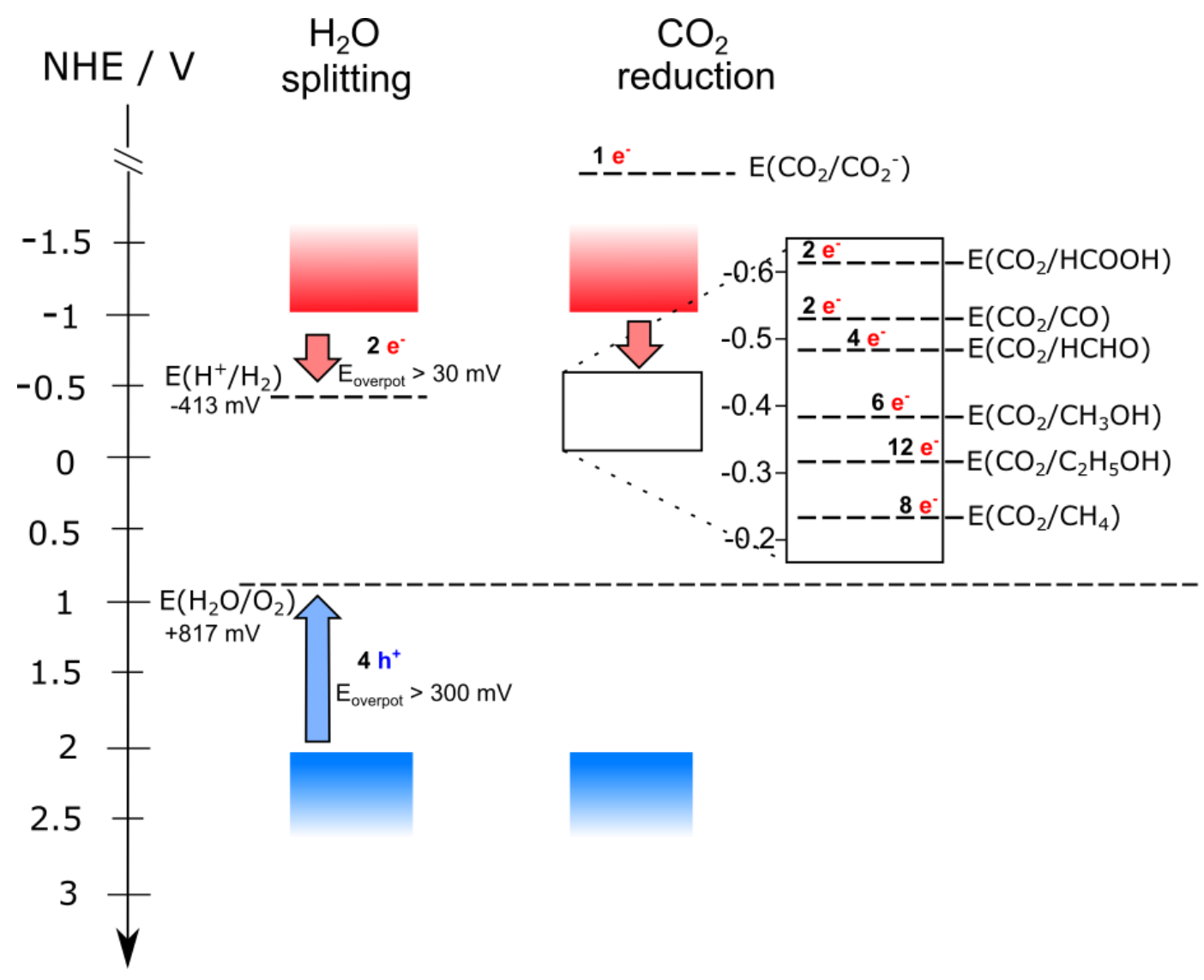

Figure 1. Energetic requirements for semiconductor photocatalysts to enable water splitting and $\mathrm{CO}_{2}$ reduction redox reactions at $\mathbf{p H ~ 7 . ~ T h e ~ c o n d u c t i o n ~ b a n d ~ ( s h o w n ~ i n ~ r e d ) ~ a n d ~ t h e ~ v a l e n c e ~ b a n d ~ ( s h o w n ~ i n ~ b l u e ) ~ n e e d ~ t o ~ s t r a d d l e ~}$ the equilibrium potentials of the respective reduction and oxidation half-reactions while also providing enough excess energy (overpotential, $\mathrm{E}_{\text {overpot}}$ ) to drive the multi-electron transfer processes at sufficiently high rates. Not only the driving force, but the stabilization of intermediates during such multi-electron transfer reactions on the catalyst surface are also important for efficient product formation and selectivity. 


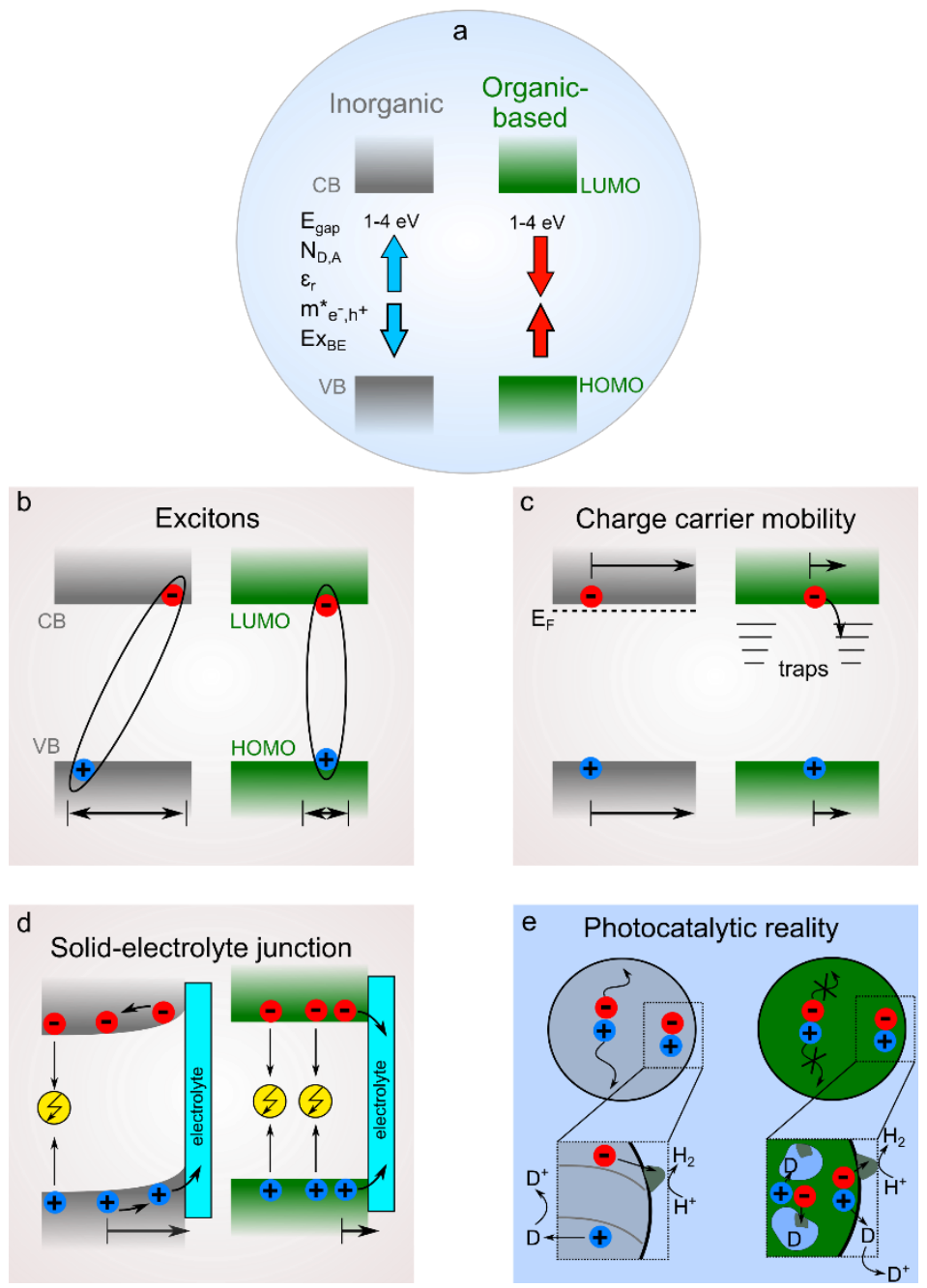

Figure 2. Differences and commonalities between inorganic and organic based semiconductor photocatalysts. (a) Crystalline inorganic semiconductors typically have high concentrations of mobile carriers $\left(\mathrm{N}_{\mathrm{D}, \mathrm{A}}\right)$ with low effective masses $\left(\mathrm{m}^{*} \mathrm{e}^{-}, \mathrm{h}^{+}\right)$, a larger dielectric constant $\left(\varepsilon_{\mathrm{r}}\right)$ and comparably low exciton binding energy $\left(\operatorname{Ex}_{\mathrm{BE}}\right)$ compared to their organic counterparts. (b) Photogenerated excitons or free charge carriers are hence more mobile in inorganic materials. (c) While charge transport in the conduction band (CB) and valence band (VB) of inorganic systems is of relatively long range, carrier mobility is limited to shorter ranges in organic systems and affected by charge carrier trapping and de-trapping. (d) The formation of a space charge layer at the solid electrolyte interface assists the transport of charge carriers towards the surface catalytic center in inorganic systems with defined doping $\left(\mathrm{N}_{\mathrm{D}, \mathrm{A}}\right)$. (e) In its absence, charge carrier generation needs to occur close to the electrolyte interface - within the diffusion limit of the generated carriers. For organic based photocatalysts therefore, the material's porosity, wettability, the particle size and the amount and type of defects are often important parameters to tune the overall photocatalytic efficiency. 
a
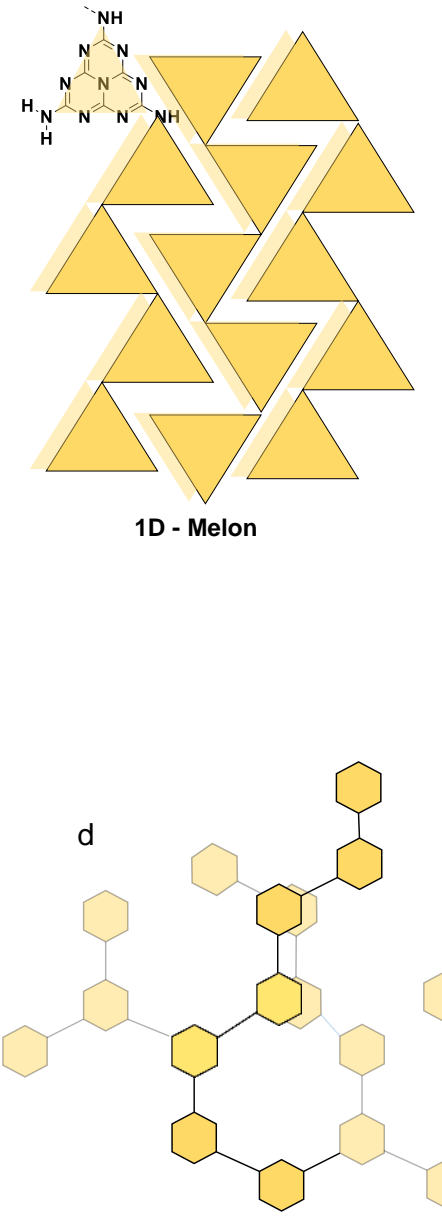

Amorphous polymer

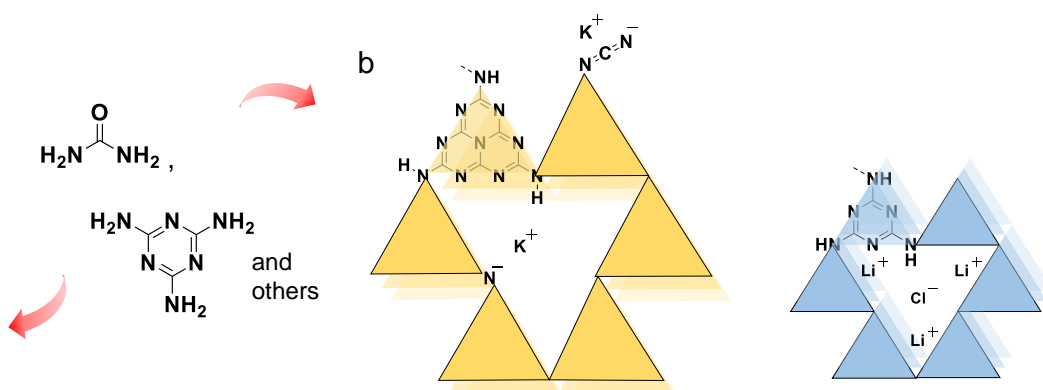

2D - K-PHI

2D $-\mathrm{PTI} / \mathrm{Li}^{+} \mathbf{X}$
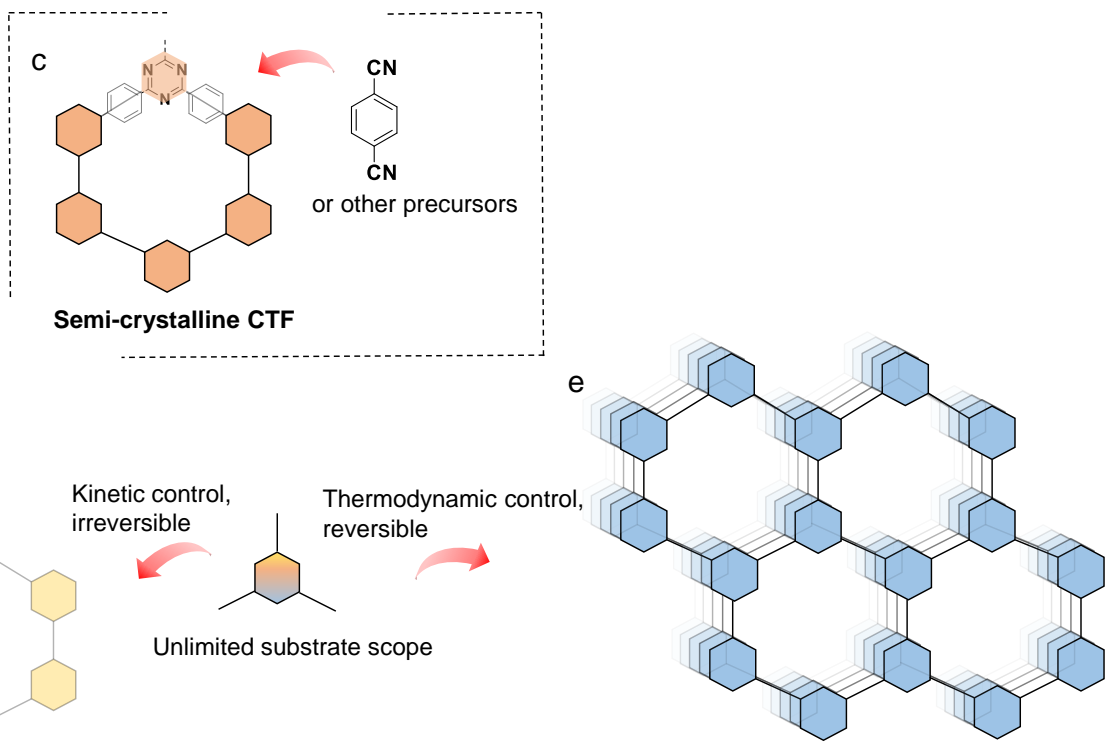

Crystalline COF

Figure 3. Synthesis and structural features of different organic polymers. (a) Melon-type carbon nitrides are heptazine based linear polymeric strands, which are connected to each other by intralayer H-bonds. The strands are further stacked in the axial direction by $\pi-\pi$ interactions. (b) Poly(triazine imide), PTI and poly(heptazine imide), PHI based carbon nitrides, on the other hand, have a true two-dimensional structure, the layers being held together by interlayer $\pi-\pi$ interactions. Carbon nitride materials are composed of heptazine and/or triazine units only and hence the substrate scope for synthesizing such polymers is very limited. (d, e) Conjugated polymers and covalent organic frameworks (COFs) are widely tunable in terms of composition. However, conjugated polymers form as amorphous products because the reaction is kinetically controlled. COFs, on the other hand, are synthesized by linking molecules using reversible reactions by thermodynamically controlled dynamic covalent chemistry, thus leading to highly crystalline products. (c) Covalent triazine frameworks (CTFs) bridge the triazine and heptazine based carbon nitrides and the $\pi$-conjugated organic polymers. These are triazine based robust semi-crystalline or amorphous polymers, which can have a high degree of compositional tunability. 
a

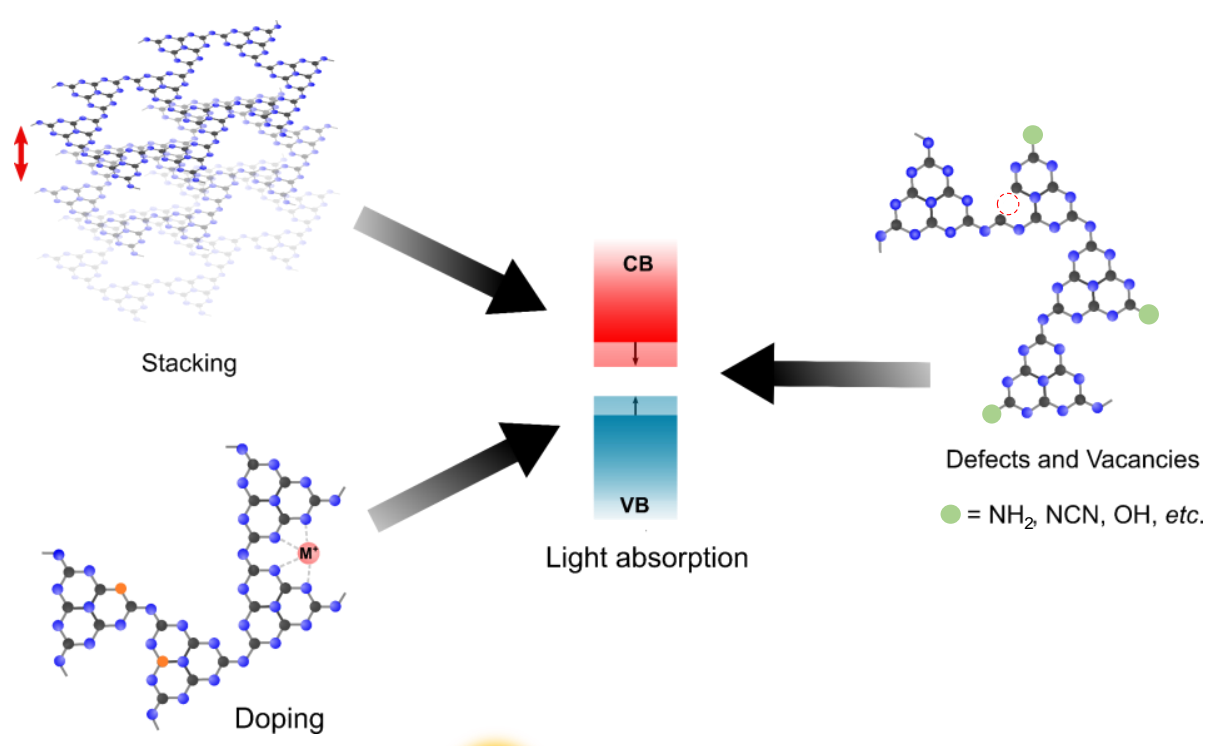

b

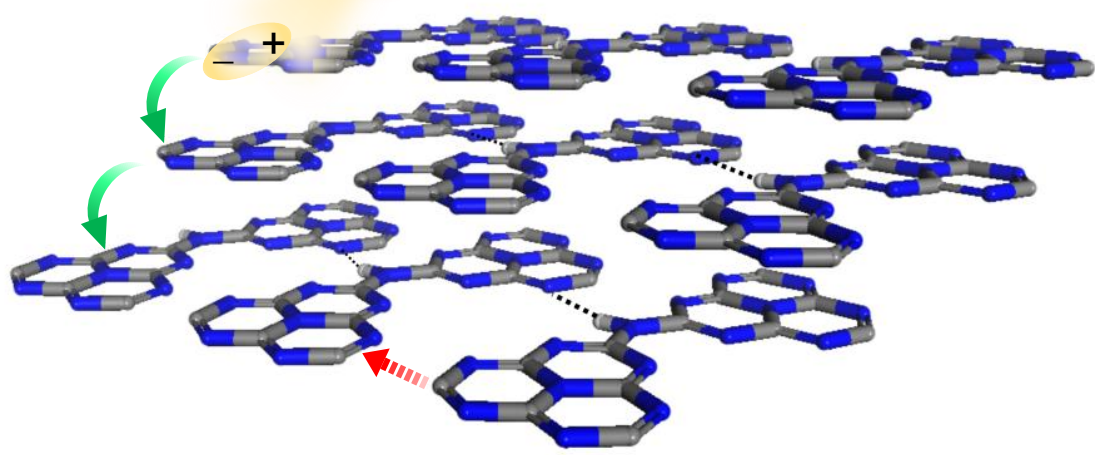

Exciton diffusion

Figure 4. Light absorption and charge carrier transport in carbon nitrides. (a) The optical band gap and hence the light absorption in carbon nitride polymers can be tuned by altering the stacking distance between the 2D layers, by doping with heteroatoms and metal ions, and by the incorporation of appropriate defect functionalities and vacancies in the structure. (b) Excited charge carrier transport in 1D carbon nitride materials is primarily interplanar. Poor electronic conjugation along the plane largely impedes intraplanar charge transport. 
a

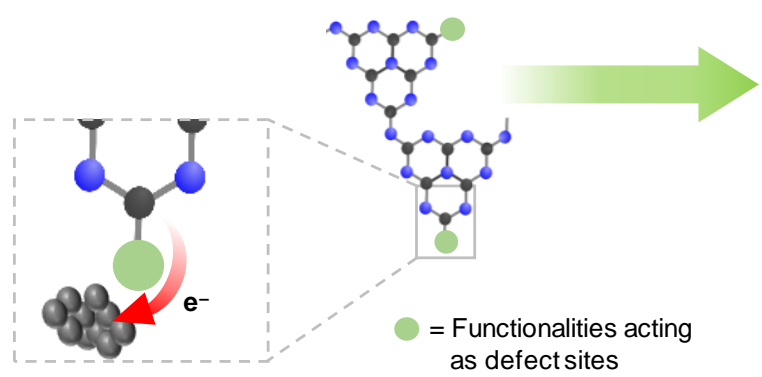

b

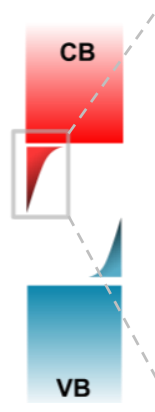

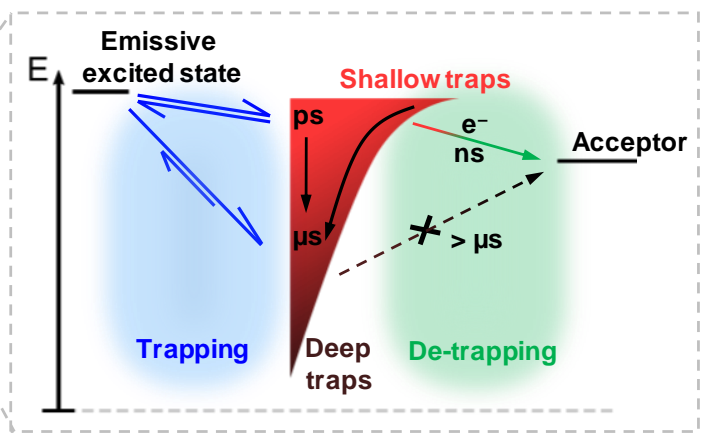

Figure 5. Defect sites for charge-transfer reaction. (a) Functional moieties like amines, urea, cyanamides in the carbon nitride structure have been suggested to act as coordination sites for metallic Pt and molecular co-catalysts, thus facilitating charge transfer to the latter, thereby increasing photocatalytic efficiency. (b) Such functional moieties are also responsible for the formation of trap states within the band gap of the material. Trapping of electrons (ps- $\mu \mathrm{s}$ ) can reduce the photocatalytic efficiency, as the deeply trapped electrons lose a large amount of energy and are unable to participate in electron transfer to the acceptor on time scales of $\mu$ s or longer due to a reduction in the thermodynamic driving force. Panel $\mathrm{b}$ is adapted from reference 52.

a

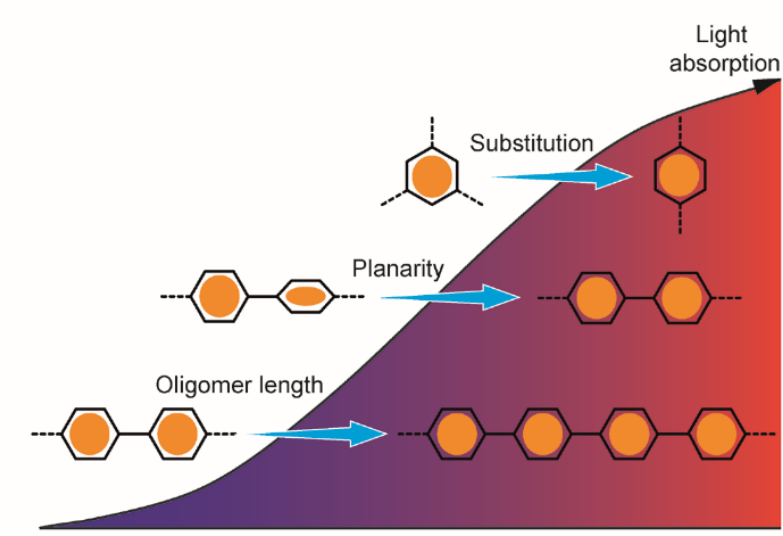

b
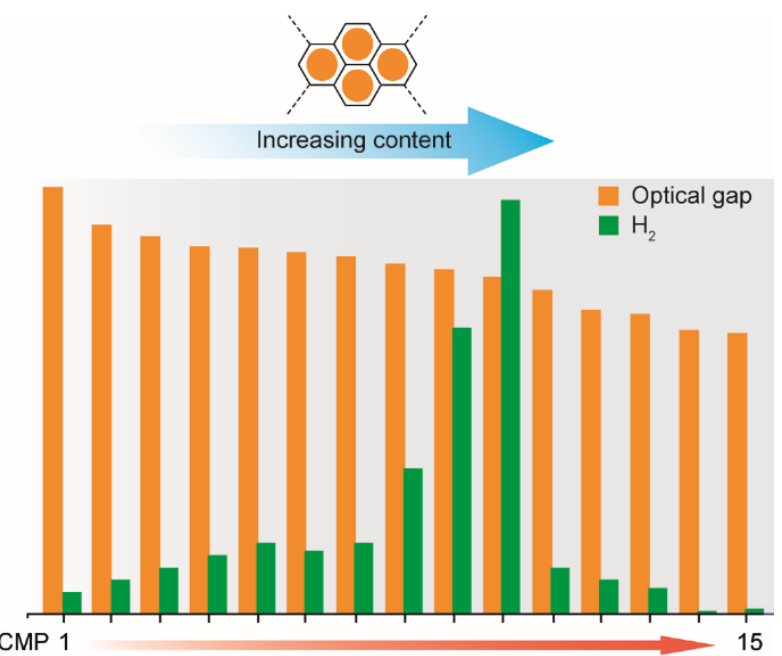

Figure 6. Molecular tuning of optoelectronics in $\boldsymbol{\pi}$-conjugated organic polymers. (a) The light absorption of such polymer photocatalysts can be enhanced by increasing the overall planarity in the structure and increasing the oligomer length, also by avoiding meta-substitution. (b) The optical band gap of pyrene containing conjugated microporous polymers can be tuned continuously by varying the pyrene content during synthesis. ${ }^{156}$ The progressively decreasing optical band gap is reflected in a corresponding increase in the photocatalytic $\mathrm{H}_{2}$ evolution activity of the polymers till a point (CP-CMP-10) when deleterious processes likely take over. Panel $b$ is adapted from reference 157. 

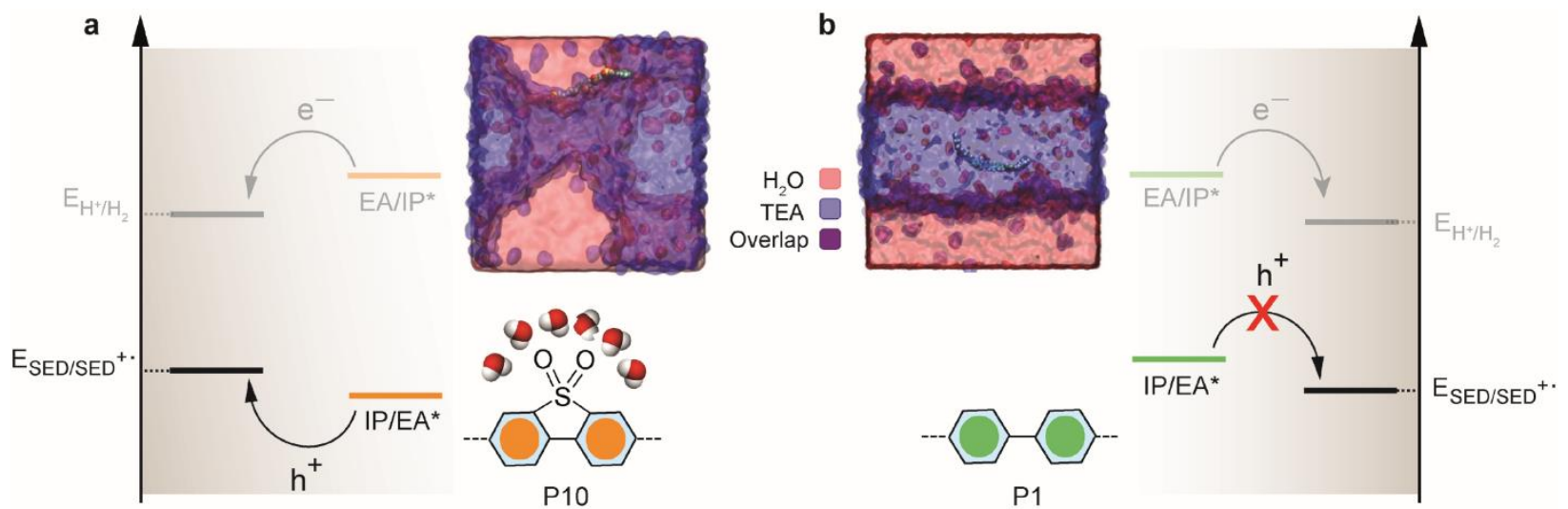

Figure 7. Modulating hydrophilicity of organic polymers for hydrogen evolution photocatalysis. The presence of sulfone groups imparts hydrophilicity to the polymer backbone as seen with atomistic molecular dynamics simulations. (a) The model oligomer for sulfone containing polymer $\mathrm{P} 10$ resides at the $\mathrm{H}_{2} \mathrm{O}$-triethylamine (TEA) interface, while (b) the model oligomer for non-polar P1 hides in the triethylamine phase. The difference in solvation significantly affects the driving force for hole transfer to triethylamine in the water:trimethylamine:methanol photocatalytic reaction mixture, making it thermodynamically downhill (a) for the sulfone containing polymers P7 and P10, and thermodynamically uphill (b) for P1 which lacks any sulfone groups. The polymers thus produce $\mathrm{H}_{2}$ in the order P10 > P7 > P1. ${ }^{39}$ SED: Sacrificial electron donor, EA: Electron affinity, IP: Ionization potential, EA*: Excited state electron affinity, IP*: Excited state ionization potential. The molecular dynamics simulations have been reproduced from ref. 40.

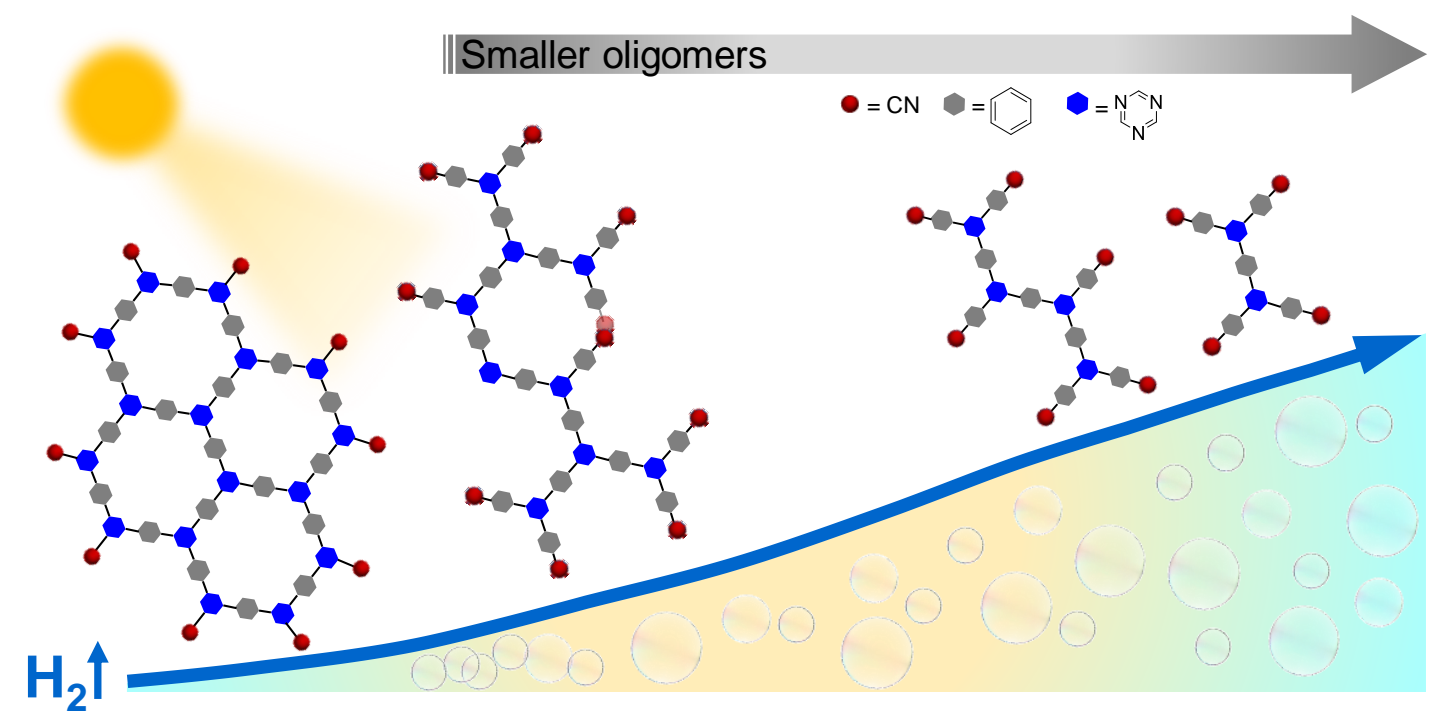

Figure 8. Oligomers as efficient photocatalysts. Increased $\mathrm{ZnCl}_{2}$ content in the low temperature ionothermal synthesis methods leads to the formation of smaller phenyltriazine oligomers (PTOs) which are more active in photocatalytic $\mathrm{H}_{2}$ evolution, suggesting a prominent role of the unreacted terminal nitrile functionalities in the process. ${ }^{192}$ 


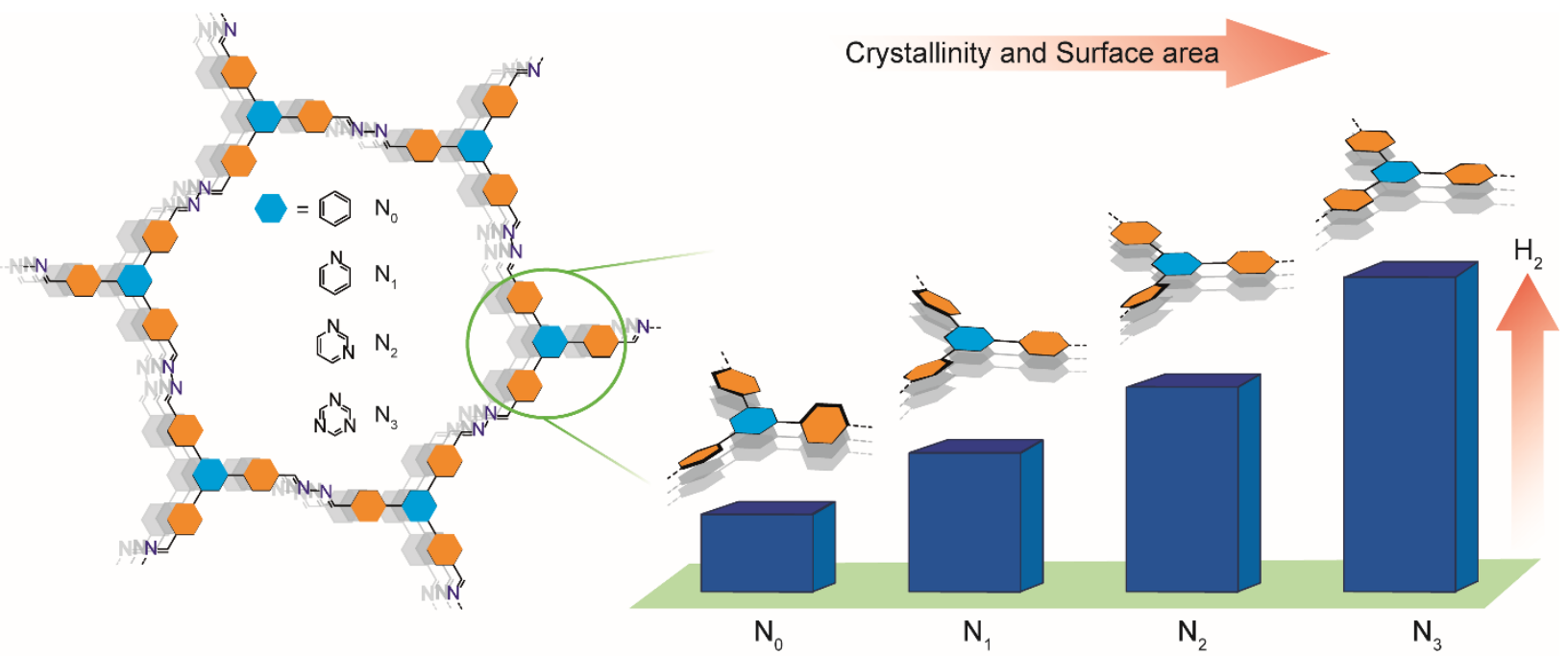

Figure 9. Manipulating crystallinity for photocatalysis by molecular level tuning. Pore view of the $\mathrm{N}_{\mathrm{x}}-\mathrm{COF}$ platform (left), where with successive isolobal substitution of $\mathrm{C}-\mathrm{H}$ units with $\mathrm{N}$ atoms the overall planarity increases with a corresponding increase in crystallinity and surface area, which contributes to increased photocatalytic $\mathrm{H}_{2}$ evolution rates up the series (right). ${ }^{213}$ The stability of the likely radical anion intermediate also increases along the series, suggesting a progressively more effective charge separation.

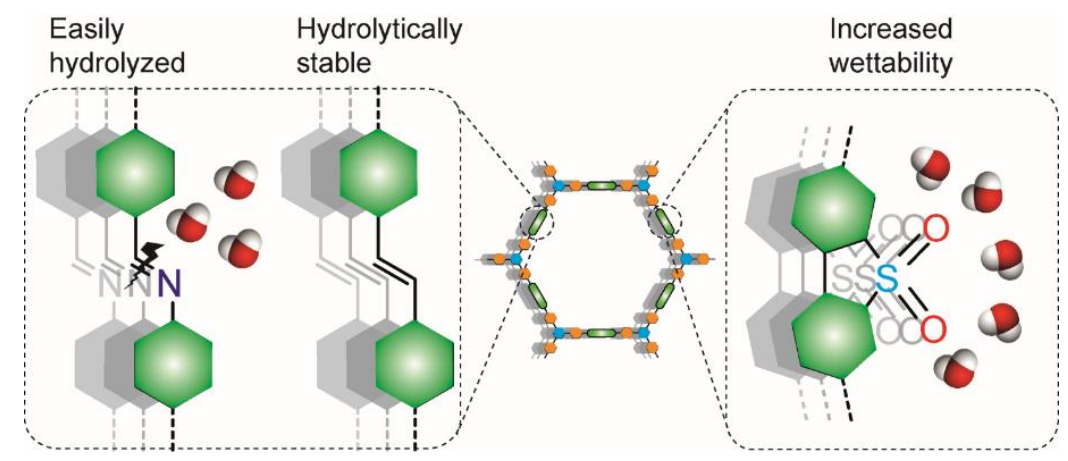

Figure 10. Linker stability and catalyst wettability as factors affecting photocatalysis with COFs. Olefin -linked COFs have significant potential as photocatalysts owing to their significantly high hydrolytic stability (left). The wettability of COF photocatalysts, which can be tuned at a molecular level by the incorporation of hydrophilic functionalities (right), is another important factor affecting the photocatalytic efficiency. 


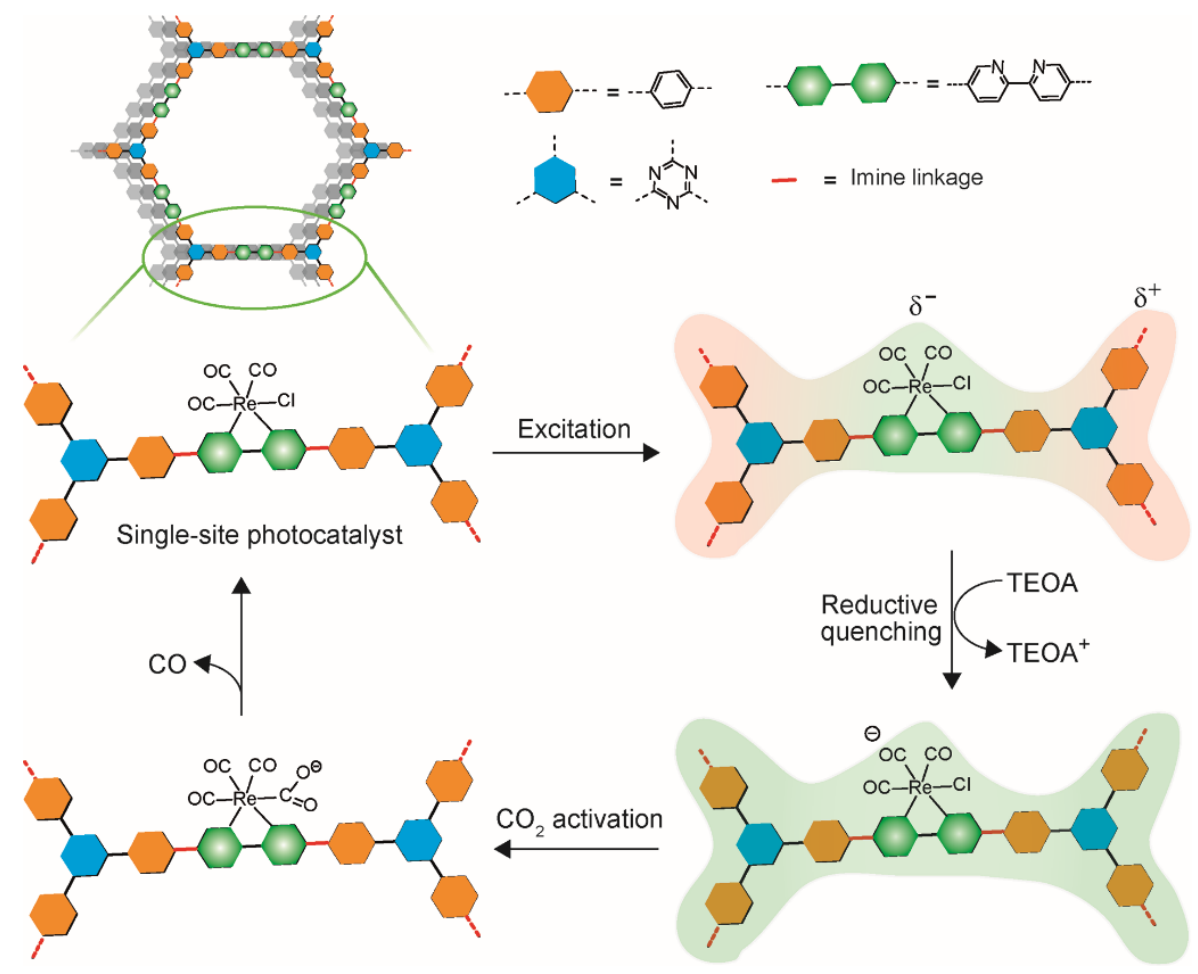

Figure 11. Single-site COF photocatalyst for $\mathrm{CO}_{2}$ photoreduction. The crystallinity and the local order in the COF backbone enables development of single site photocatalysts like Re-COF. Illumination results in a charge separated state with electrons partially located on the $\operatorname{Re}($ bpy $)(\mathrm{CO})_{3} \mathrm{Cl}$ moiety. Reductive quenching by TEOA and consequent $\mathrm{CO}_{2}$ activation by the Re complex follows, ultimately liberating $\mathrm{CO}$ and regenerating the photocatalyst. Adapted from reference 249 .

\section{Acknowledgements}

Financial support by an ERC Starting Grant (project COF Leaf, Grant Number 639233), the Deutsche Forschungsgemeinschaft (DFG, German Research Foundation) - Project number 358283783 - SFB 1333, the Max Planck Society, the Cluster of Excellence e-conversion, and the Center for Nanoscience (CeNS) is gratefully acknowledged.

\section{Author contributions}

T.B., F.P. and J.K. wrote the manuscript along with contributions from B.V.L. and B.P.B. All authors read and commented on the final manuscript.

\section{Competing interests statement}

The authors declare no competing interests. 


\section{References}

1 Balzani, V., Bergamini, G. \& Ceroni, P. Photochemistry and photocatalysis. Rend. Fis. Acc. Lincei 28, 125-142 (2017).

2 Ravelli, D., Dondi, D., Fagnoni, M. \& Albini, A. Photocatalysis. A multi-faceted concept for green chemistry. Chem. Soc. Rev. 38, 1999-2011 (2009).

3 Fagnoni, M., Dondi, D., Ravelli, D. \& Albini, A. Photocatalysis for the Formation of the C-C Bond. Chem. Rev. 107, 2725-2756 (2007).

4 Osterloh, F. E. Photocatalysis versus Photosynthesis: A Sensitivity Analysis of Devices for Solar Energy Conversion and Chemical Transformations. ACS Energy Lett. 2, 445-453 (2017).

5 Prier, C. K., Rankic, D. A. \& MacMillan, D. W. C. Visible Light Photoredox Catalysis with Transition Metal Complexes: Applications in Organic Synthesis. Chem. Rev. 113, 5322-5363 (2013).

6 McAtee, R. C., McClain, E. J. \& Stephenson, C. R. J. Illuminating Photoredox Catalysis. Trends Chem. 1, 111-125 (2019).

7 Fujishima, A. \& Honda, K. Electrochemical Photolysis of Water at a Semiconductor Electrode. Nature 238, 37-38 (1972).

8 Xing, J., Fang, W. Q., Zhao, H. J. \& Yang, H. G. Inorganic Photocatalysts for Overall Water Splitting. Chem. Asian J. 7, 642-657 (2012).

9 Osterloh, F. E. Inorganic nanostructures for photoelectrochemical and photocatalytic water splitting. Chem. Soc. Rev. 42, 2294-2320 (2013).

10 Wang, Q. \& Domen, K. Particulate Photocatalysts for Light-Driven Water Splitting: Mechanisms, Challenges, and Design Strategies. Chem. Rev. 120, 919-985 (2020).

11 Du, P. \& Eisenberg, R. Catalysts made of earth-abundant elements (Co, Ni, Fe) for water splitting: Recent progress and future challenges. Energy Environ. Sci. 5, 6012-6021 (2012).

12 Liu, S., Lei, Y.-J., Xin, Z.-J., Lu, Y.-B. \& Wang, H.-Y. Water splitting based on homogeneous copper molecular catalysts. J. Photochem. Photobiol. A Chem. 355, 141-151 (2018).

13 Thoi, V. S., Sun, Y., Long, J. R. \& Chang, C. J. Complexes of earth-abundant metals for catalytic electrochemical hydrogen generation under aqueous conditions. Chem. Soc. Rev. 42, 2388-2400 (2013).

14 Vyas, V. S., Lau, V. W.-h. \& Lotsch, B. V. Soft Photocatalysis: Organic Polymers for Solar Fuel Production. Chem. Mater. 28, 5191-5204 (2016).

15 Wang, Y. et al. Current understanding and challenges of solar-driven hydrogen generation using polymeric photocatalysts. Nat. Energy 4, 746-760 (2019).

16 Takanabe, K. Photocatalytic Water Splitting: Quantitative Approaches toward Photocatalyst by Design. ACS Catal. 7, 8006-8022 (2017).

17 Chen, Z., Dinh, H.N. \& Miller, E. Photoelectrochemical Water Splitting. (Springer, New York, NY, 2013).

18 Hagfeldt, A., Boschloo, G., Sun, L., Kloo, L. \& Pettersson, H. Dye-Sensitized Solar Cells. Chem. Rev. 110, 6595-6663 (2010).

19 Habisreutinger, S. N., Schmidt-Mende, L. \& Stolarczyk, J. K. Photocatalytic Reduction of $\mathrm{CO}_{2}$ on $\mathrm{TiO}_{2}$ and Other Semiconductors. Angew. Chem. Int. Ed. 52, 7372-7408 (2013).

20 Qiao, J., Liu, Y., Hong, F. \& Zhang, J. A review of catalysts for the electroreduction of carbon dioxide to produce low-carbon fuels. Chem. Soc. Rev. 43, 631-675 (2014).

21 Bisquert, J., Cendula, P., Bertoluzzi, L. \& Gimenez, S. Energy Diagram of Semiconductor/Electrolyte Junctions. J. Phys. Chem. Lett. 5, 205-207 (2014).

22 Williams, F. \& Nozik, A. J. Solid-state perspectives of the photoelectrochemistry of semiconductor-electrolyte junctions. Nature 312, 21-27 (1984).

23 Bard, A. J., Bocarsly, A. B., Fan, F. R. F., Walton, E. G. \& Wrighton, M. S. The concept of Fermi level pinning at semiconductor/liquid junctions. Consequences for energy conversion efficiency 
and selection of useful solution redox couples in solar devices. J. Am. Chem. Soc. 102, 3671-3677 (1980).

24 Klein, A. Energy band alignment at interfaces of semiconducting oxides: A review of experimental determination using photoelectron spectroscopy and comparison with theoretical predictions by the electron affinity rule, charge neutrality levels, and the common anion rule. Thin Solid Films $\mathbf{5 2 0}$, 3721-3728 (2012).

25 Tan, H. L., Abdi, F. F. \& Ng, Y. H. Heterogeneous photocatalysts: an overview of classic and modern approaches for optical, electronic, and charge dynamics evaluation. Chem. Soc. Rev. 48, 1255-1271 (2019).

26 Xu, P., Milstein, T. J. \& Mallouk, T. E. Flat-Band Potentials of Molecularly Thin Metal Oxide Nanosheets. ACS Appl. Mater. Interfaces 8, 11539-11547 (2016).

27 Noda, Y. et al. Directional Charge Transport in Layered Two-Dimensional Triazine-Based Graphitic Carbon Nitride. Angew. Chem. Int. Ed. 58, 9394-9398 (2019).

28 Le Bahers, T., Rérat, M. \& Sautet, P. Semiconductors Used in Photovoltaic and Photocatalytic Devices: Assessing Fundamental Properties from DFT. J. Phys. Chem. C 118, 5997-6008 (2014).

29 Park, H., Kim, H.-i., Moon, G.-h. \& Choi, W. Photoinduced charge transfer processes in solar photocatalysis based on modified $\mathrm{TiO}_{2}$. Energy Environ. Sci. 9, 411-433 (2016).

30 Clarke, T. M. \& Durrant, J. R. Charge Photogeneration in Organic Solar Cells. Chem. Rev. 110, 6736-6767 (2010).

31 Guiglion, P., Butchosa, C. \& Zwijnenburg, M. A. Polymer Photocatalysts for Water Splitting: Insights from Computational Modeling. Macromol. Chem. Phys. 217, 344-353 (2016).

32 Puschnig, P. \& Ambrosch-Draxl, C. Excitons in organic semiconductors. C. R. Physique 10, 504513 (2009).

33 Hummer, K., Puschnig, P., Sagmeister, S. \& Ambrosch-Draxl, C. Ab-Initio Study on the Exciton Binding Energies in Organic Semiconductors. Mod. Phys. Lett. B 20, 261-280 (2006).

34 Melissen, S., Le Bahers, T., Steinmann, S. N. \& Sautet, P. Relationship between Carbon Nitride Structure and Exciton Binding Energies: A DFT Perspective. J. Phys. Chem. C 119, 25188-25196 (2015).

35 Zhang, G. et al. Optimizing Optical Absorption, Exciton Dissociation, and Charge Transfer of a Polymeric Carbon Nitride with Ultrahigh Solar Hydrogen Production Activity. Angew. Chem. 56, 13445-13449 (2017).

36 Lin, Z. \& Wang, X. Nanostructure Engineering and Doping of Conjugated Carbon Nitride Semiconductors for Hydrogen Photosynthesis. Angew. Chem. Int. Ed. 52, 1735-1738 (2013).

37 Bässler, H. \& Köhler, A. Charge Transport in Organic Semiconductors. In: Unimolecular and Supramolecular Electronics I. Vol. 312 1-65 (Springer, Berlin, Heidelberg, 2011).

38 Merschjann, C. et al. Complementing Graphenes: 1D Interplanar Charge Transport in Polymeric Graphitic Carbon Nitrides. Adv. Mater. 27, 7993-7999 (2015).

39 Rahman, M. Z. \& Mullins, C. B. Understanding Charge Transport in Carbon Nitride for Enhanced Photocatalytic Solar Fuel Production. Acc. Chem. Res. 52, 248-257 (2019).

40 Sachs, M. et al. Understanding structure-activity relationships in linear polymer photocatalysts for hydrogen evolution. Nat. Commun. 9, 4968 (2018).

41 Medina, D. D. et al. Oriented Thin Films of a Benzodithiophene Covalent Organic Framework. ACS Nano 8, 4042-4052 (2014).

42 Pelzer, K. M. \& Darling, S. B. Charge generation in organic photovoltaics: a review of theory and computation. Mol. Syst. Des. Eng. 1, 10-24 (2016).

43 Botiz, I., Schaller, R. D., Verduzco, R. \& Darling, S. B. Optoelectronic Properties and Charge Transfer in Donor-Acceptor All-Conjugated Diblock Copolymers. J. Phys. Chem. C 115, 92609266 (2011).

44 Zhou, W. \& Fu, H. Defect-mediated electron-hole separation in semiconductor photocatalysis. Inorg. Chem. Front. 5, 1240-1254 (2018). 
Lau, V. W.-h. et al. Rational design of carbon nitride photocatalysts by identification of cyanamide defects as catalytically relevant sites. Nat. Commun. 7, 12165 (2016).

46 Zhang, J., Zhang, M., Sun, R.-Q. \& Wang, X. A Facile Band Alignment of Polymeric Carbon Nitride Semiconductors to Construct Isotype Heterojunctions. Angew. Chem. Int. Ed. 51, 1014510149 (2012).

47 Heremans, P., Cheyns, D. \& Rand, B. P. Strategies for Increasing the Efficiency of Heterojunction Organic Solar Cells: Material Selection and Device Architecture. Acc. Chem. Res. 42, 1740-1747 (2009).

48 Banerjee, T., Gottschling, K., Savasci, G., Ochsenfeld, C. \& Lotsch, B. V. H ${ }_{2}$ Evolution with Covalent Organic Framework Photocatalysts. ACS Energy Lett. 3, 400-409 (2018).

49 Knupfer, M. Exciton binding energies in organic semiconductors. Appl. Phys. A 77, 623-626 (2003).

50 Tamai, Y., Ohkita, H., Benten, H. \& Ito, S. Exciton Diffusion in Conjugated Polymers: From Fundamental Understanding to Improvement in Photovoltaic Conversion Efficiency. J. Phys. Chem. Lett. 6, 3417-3428 (2015).

51 Gregg, B. A. Charged defects in soft semiconductors and their influence on organic photovoltaics. Soft Matter 5, 2985-2989 (2009).

52 Godin, R., Wang, Y., Zwijnenburg, M. A., Tang, J. \& Durrant, J. R. Time-Resolved Spectroscopic Investigation of Charge Trapping in Carbon Nitrides Photocatalysts for Hydrogen Generation. $J$. Amer. Chem. Soc. 139, 5216-5224 (2017).

Defect sites contribute to the formation of electron trap states in melon and charge carrier trapping can reduce the efficiency of surface photocatalytic reactions.

53 Lau, V. W.-h. et al. Urea-Modified Carbon Nitrides: Enhancing Photocatalytic Hydrogen Evolution by Rational Defect Engineering. Adv. Energy Mater. 7, 1602251 (2017).

54 Steinmann, S. N., Melissen, S. T. A. G., Le Bahers, T. \& Sautet, P. Challenges in calculating the bandgap of triazine-based carbon nitride structures. J. Mater. Chem. A 5, 5115-5122 (2017).

55 Jones, E. D. Control of Semiconductor Conductivity by Doping. In: Electronic Materials. (Springer, Boston, MA, 1991).

56 Yin, W.-J., Shi, T. \& Yan, Y. Unusual defect physics in $\mathrm{CH}_{3} \mathrm{NH}_{3} \mathrm{PbI}_{3}$ perovskite solar cell absorber. Appl. Phys. Lett. 104, 063903 (2014).

57 Ball, J. M. \& Petrozza, A. Defects in perovskite-halides and their effects in solar cells. Nat. Energy 1, 16149 (2016).

58 Luo, Y., Yan, Y., Zheng, S., Xue, H. \& Pang, H. Graphitic carbon nitride based materials for electrochemical energy storage. J. Mater. Chem. A 7, 901-924 (2019).

59 Wenderott, J. K., Dong, B. X. \& Green, P. F. Band bending in conjugated polymer films: role of morphology and implications for bulk charge transport characteristics. J. Mater. Chem. C 5, 74467451 (2017).

60 Zhang, Z. \& Yates, J. T. Band Bending in Semiconductors: Chemical and Physical Consequences at Surfaces and Interfaces. Chem. Rev. 112, 5520-5551 (2012).

61 Hagfeldt, A. \& Graetzel, M. Light-Induced Redox Reactions in Nanocrystalline Systems. Chem. Rev. 95, 49-68 (1995).

62 Kirchartz, T. et al. Sensitivity of the Mott-Schottky Analysis in Organic Solar Cells. J. Phys. Chem. C 116, 7672-7680 (2012).

63 Hankin, A., Bedoya-Lora, F. E., Alexander, J. C., Regoutz, A. \& Kelsall, G. H. Flat band potential determination: avoiding the pitfalls. J. Mater. Chem. A 7, 26162-26176 (2019).

64 Almora, O., Aranda, C., Mas-Marzá, E. \& Garcia-Belmonte, G. On Mott-Schottky analysis interpretation of capacitance measurements in organometal perovskite solar cells. Appl. Phys. Lett. 109, 173903 (2016).

65 Kessler, F. K. et al. Functional carbon nitride materials - design strategies for electrochemical devices. Nat. Rev. Mater. 2, 17030 (2017). 

Based Photocatalysts for Artificial Photosynthesis and Environmental Remediation: Are We a Step Closer To Achieving Sustainability? Chem. Rev. 116, 7159-7329 (2016).

67 Zheng, Y., Lin, L., Wang, B. \& Wang, X. Graphitic Carbon Nitride Polymers toward Sustainable Photoredox Catalysis. Angew. Chem. Int. Ed. 54, 12868-12884 (2015).

68 Safaei, J. et al. Graphitic carbon nitride $\left(\mathrm{g}-\mathrm{C}_{3} \mathrm{~N}_{4}\right)$ electrodes for energy conversion and storage: a review on photoelectrochemical water splitting, solar cells and supercapacitors. J. Mater. Chem. A 6, 22346-22380 (2018).

69 Yang, Y., Wang, S., Li, Y., Wang, J. \& Wang, L. Strategies for Efficient Solar Water Splitting Using Carbon Nitride. Chem. Asian J. 12, 1421-1434 (2017).

70 Liao, G. et al. Semiconductor polymeric graphitic carbon nitride photocatalysts: the "holy grail" for the photocatalytic hydrogen evolution reaction under visible light. Energy Environ. Sci. 12, 2080-2147 (2019).

71 Wang, X. et al. A metal-free polymeric photocatalyst for hydrogen production from water under visible light. Nat. Mater. 8, 76-80 (2009).

\section{First report of photocatalytic $\mathrm{H}_{2}$ and $\mathrm{O}_{2}$ evolution with melon-type carbon nitrides.}

72 Maeda, K. et al. Photocatalytic Activities of Graphitic Carbon Nitride Powder for Water Reduction and Oxidation under Visible Light. J. Phys. Chem. C 113, 4940-4947 (2009).

73 Lotsch, B. V. et al. Unmasking Melon by a Complementary Approach Employing Electron Diffraction, Solid-State NMR Spectroscopy, and Theoretical Calculations-Structural Characterization of a Carbon Nitride Polymer. Chem. Eur. J. 13, 4969-4980 (2007).

Seyfarth, L., Seyfarth, J., Lotsch, B. V., Schnick, W. \& Senker, J. Tackling the stacking disorder of melon-structure elucidation in a semicrystalline material. Phys. Chem. Chem. Phys. 12, 22272237 (2010).

75 Schwinghammer, K. et al. Triazine-based Carbon Nitrides for Visible-Light-Driven Hydrogen Evolution. Angew. Chem. Int. Ed. 52, 2435-2439 (2013).

76 Schlomberg, H. et al. Structural Insights into Poly(Heptazine Imides): A Light-Storing Carbon Nitride Material for Dark Photocatalysis. Chem. Mater. 31, 7478-7486 (2019).

77 Wirnhier, E. et al. Poly(triazine imide) with Intercalation of Lithium and Chloride Ions $\left[\left(\mathrm{C}_{3} \mathrm{~N}_{3}\right)_{2}\left(\mathrm{NH}_{\mathrm{x}} \mathrm{Li}_{1-\mathrm{x}}\right)_{3} \cdot \mathrm{LiCl}\right]$ : A Crystalline 2D Carbon Nitride Network. Chem. Eur. J. 17, 3213-3221 (2011).

78 Ham, Y., Maeda, K., Cha, D., Takanabe, K. \& Domen, K. Synthesis and Photocatalytic Activity of Poly(triazine imide). Chem. Asian J. 8, 218-224 (2013).

79 Kessler, F. K. \& Schnick, W. From Heptazines to Triazines - On the Formation of Poly(triazine imide). Z. Anorg. Allg. Chem. 645, 857-862 (2019).

80 Bhunia, M. K., Yamauchi, K. \& Takanabe, K. Harvesting Solar Light with Crystalline Carbon Nitrides for Efficient Photocatalytic Hydrogen Evolution. Angew. Chem. Int. Ed. 53, 11001-11005 (2014).

81 Chen, Z. et al. "The Easier the Better" Preparation of Efficient Photocatalysts-Metastable Poly(heptazine imide) Salts. Adv. Mater. 29, 1700555 (2017).

82 Zhang, G. et al. Tailoring the Grain Boundary Chemistry of Polymeric Carbon Nitride for Enhanced Solar Hydrogen Production and $\mathrm{CO}_{2}$ Reduction. Angew. Chem. Int. Ed. 58, 3433-3437 (2019).

83 Savateev, A., Pronkin, S., Willinger, M. G., Antonietti, M. \& Dontsova, D. Towards Organic Zeolites and Inclusion Catalysts: Heptazine Imide Salts Can Exchange Metal Cations in the Solid State. Chem. Asian J. 12, 1517-1522 (2017).

84 Savateev, A. et al. Potassium Poly(heptazine imides) from Aminotetrazoles: Shifting Band Gaps of Carbon Nitride-like Materials for More Efficient Solar Hydrogen and Oxygen Evolution. ChemCatChem 9, 167-174 (2017).

85 Gao, H. et al. Towards efficient solar hydrogen production by intercalated carbon nitride photocatalyst. Phys. Chem. Chem. Phys. 15, 18077-18084 (2013). 

Phys. Chem. C 117, 8806-8812 (2013).

87 Savateev, A., Kurpil, B., Mishchenko, A., Zhang, G. \& Antonietti, M. A “waiting” carbon nitride radical anion: a charge storage material and key intermediate in direct $\mathrm{C}-\mathrm{H}$ thiolation of methylarenes using elemental sulfur as the "S"-source. Chem. Sci. 9, 3584-3591 (2018). oxadiazoles catalyzed by heterogeneous visible-light-active carbon nitride semiconductor. Appl. Catal. B 228, 97-102 (2018).

89 Savateev, A., Dontsova, D., Kurpil, B. \& Antonietti, M. Highly crystalline poly(heptazine imides) by mechanochemical synthesis for photooxidation of various organic substrates using an intriguing electron acceptor - Elemental sulfur. J. Catal. 350, 203-211 (2017).

90 Kurpil, B. et al. Carbon nitride photocatalyzes regioselective aminium radical addition to the carbonyl bond and yields N-fused pyrroles. Nat. Commun. 10, 945 (2019).

91 Kurpil, B. et al. Hexaazatriphenylene doped carbon nitrides-Biomimetic photocatalyst with superior oxidation power. Appl. Catal. B 217, 622-628 (2017).

92 Mazzanti, S., Kurpil, B., Pieber, B., Antonietti, M. \& Savateev, A. Dichloromethylation of enones by carbon nitride photocatalysis. Nat. Commun. 11, 1387 (2020).

93 Merschjann, C. et al. Photophysics of polymeric carbon nitride: An optical quasimonomer. Phys. Rev. B 87, 205204 (2013).

94 Ullah, N., Chen, S., Zhao, Y. \& Zhang, R. Photoinduced Water-Heptazine Electron-Driven Proton Transfer: Perspective for Water Splitting with g- $\mathrm{C}_{3} \mathrm{~N}_{4}$. J. Phys. Chem. Lett. 10, 4310-4316 (2019). Ehrmaier, J., Karsili, T. N. V., Sobolewski, A. L. \& Domcke, W. Mechanism of Photocatalytic Water Splitting with Graphitic Carbon Nitride: Photochemistry of the Heptazine-Water Complex. J. Phys. Chem. A 121, 4754-4764 (2017).

96 Wei, W. \& Jacob, T. Strong excitonic effects in the optical properties of graphitic carbon nitride $g$ $\mathrm{C}_{3} \mathrm{~N}_{4}$ from first principles. Phys. Rev. B 87, 085202 (2013).

97 Zhang, X. et al. Enhanced Photoresponsive Ultrathin Graphitic-Phase $\mathrm{C}_{3} \mathrm{~N}_{4}$ Nanosheets for Bioimaging. J. Am. Chem. Soc. 135, 18-21 (2013).

98 Wang, H. et al. Insights into the excitonic processes in polymeric photocatalysts. Chem. Sci. 8, 4087-4092 (2017).

99 Ehrmaier, J. et al. Singlet-Triplet Inversion in Heptazine and in Polymeric Carbon Nitrides. $J$. Phys. Chem. A 123, 8099-8108 (2019).

100 Wilson, J. A. \& Yoffe, A. D. The transition metal dichalcogenides discussion and interpretation of the observed optical, electrical and structural properties. Adv. Phys. 18, 193-335 (1969).

101 Krishnan, K. S. \& Ganguli, N. Large Anisotropy of the Electrical Conductivity of Graphite. Nature 144, 667-667 (1939).

102 Lau, V. W.-h. et al. Dark Photocatalysis: Storage of Solar Energy in Carbon Nitride for TimeDelayed Hydrogen Generation. Angew. Chem. Int. Ed. 56, 510-514 (2017).

Photogenerated electrons are trapped in a poly(heptazine imide) photocatalyst, enabling timedelayed "dark photocatalysis".

103 Markushyna, Y. et al. Green radicals of potassium poly(heptazine imide) using light and benzylamine. J. Mater. Chem. A 7, 24771-24775 (2019).

104 Podjaski, F., Kröger, J. \& Lotsch, B. V. Toward an Aqueous Solar Battery: Direct Electrochemical Storage of Solar Energy in Carbon Nitrides. Adv. Mater. 30, 1705477 (2018).

105 Yang, W. et al. Electron Accumulation Induces Efficiency Bottleneck for Hydrogen Production in Carbon Nitride Photocatalysts. J. Amer. Chem. Soc. 141, 11219-11229 (2019).

106 Chen, Z., Zhang, Q. \& Luo, Y. Experimental Identification of Ultrafast Reverse Hole Transfer at the Interface of the Photoexcited Methanol/Graphitic Carbon Nitride System. Angew. Chem. Int. Ed. 57, 5320-5324 (2018).

107 Schwinghammer, K. et al. Crystalline Carbon Nitride Nanosheets for Improved Visible-Light Hydrogen Evolution. J. Am. Chem. Soc. 136, 1730-1733 (2014). 
108 Yang, S. et al. Exfoliated Graphitic Carbon Nitride Nanosheets as Efficient Catalysts for Hydrogen Evolution Under Visible Light. Adv. Mater. 25, 2452-2456 (2013).

109 Li, Y. et al. Targeted Exfoliation and Reassembly of Polymeric Carbon Nitride for Efficient Photocatalysis. Adv. Funct. Mater. 29, 1901024 (2019).

110 Bojdys, M. J. et al. Exfoliation of Crystalline 2D Carbon Nitride: Thin Sheets, Scrolls and Bundles via Mechanical and Chemical Routes. Macromol. Rapid Commun. 34, 850-854 (2013).

111 Xu, J., Zhang, L., Shi, R. \& Zhu, Y. Chemical exfoliation of graphitic carbon nitride for efficient heterogeneous photocatalysis. J. Mater. Chem. A 1, 14766-14772 (2013).

112 Zhang, J., Zhang, M., Yang, C. \& Wang, X. Nanospherical Carbon Nitride Frameworks with Sharp Edges Accelerating Charge Collection and Separation at a Soft Photocatalytic Interface. Adv. Mater. 26, 4121-4126 (2014).

113 Sun, J. et al. Bioinspired hollow semiconductor nanospheres as photosynthetic nanoparticles. Nat. Commun. 3, 1139 (2012).

114 Yang, Z., Zhang, Y. \& Schnepp, Z. Soft and hard templating of graphitic carbon nitride. J. Mater. Chem. A 3, 14081-14092 (2015).

115 Wang, Y., Zhang, J., Wang, X., Antonietti, M. \& Li, H. Boron- and Fluorine-Containing Mesoporous Carbon Nitride Polymers: Metal-Free Catalysts for Cyclohexane Oxidation. Angew. Chem. Int. Ed. 49, 3356-3359 (2010).

116 Jun, Y.-S. et al. Three-Dimensional Macroscopic Assemblies of Low-Dimensional Carbon Nitrides for Enhanced Hydrogen Evolution. Angew. Chem. Int. Ed. 52, 11083-11087 (2013).

117 Jun, Y.-S. et al. From Melamine-Cyanuric Acid Supramolecular Aggregates to Carbon Nitride Hollow Spheres. Adv. Funct. Mater. 23, 3661-3667 (2013).

118 Niu, P., Zhang, L., Liu, G. \& Cheng, H.-M. Graphene-Like Carbon Nitride Nanosheets for Improved Photocatalytic Activities. Adv. Funct. Mater. 22, 4763-4770 (2012).

119 Zhang, J.-H., Wei, M.-J., Wei, Z.-W., Pan, M. \& Su, C.-Y. Ultrathin Graphitic Carbon Nitride Nanosheets for Photocatalytic Hydrogen Evolution. ACS Appl. Nano Mater. 3, 1010-1018 (2020).

120 Wahab, M. A. et al. Nanoconfined Synthesis of Nitrogen-Rich Metal-Free Mesoporous Carbon Nitride Electrocatalyst for the Oxygen Evolution Reaction. ACS Appl. Energy Mater. 3, 1439-1447 (2020).

121 Kang, Y. et al. Selective Breaking of Hydrogen Bonds of Layered Carbon Nitride for Visible Light Photocatalysis. Adv. Mater. 28, 6471-6477 (2016).

122 Lau, V. W.-h. et al. Low-Molecular-Weight Carbon Nitrides for Solar Hydrogen Evolution. J. Am. Chem. Soc. 137, 1064-1072 (2015).

123 Corp, K. L. \& Schlenker, C. W. Ultrafast Spectroscopy Reveals Electron-Transfer Cascade That Improves Hydrogen Evolution with Carbon Nitride Photocatalysts. J. Am. Chem. Soc. 139, 79047912 (2017).

124 Xue, J., Fujitsuka, M. \& Majima, T. The role of nitrogen defects in graphitic carbon nitride for visible-light-driven hydrogen evolution. Phys. Chem. Chem. Phys. 21, 2318-2324 (2019).

125 Zhang, N., Gao, C. \& Xiong, Y. Defect engineering: A versatile tool for tuning the activation of key molecules in photocatalytic reactions. J. Energy Chem. 37, 43-57 (2019).

126 Wang, Q., Lei, Y., Wang, D. \& Li, Y. Defect engineering in earth-abundant electrocatalysts for $\mathrm{CO}_{2}$ and $\mathrm{N}_{2}$ reduction. Energy Environ. Sci. 12, 1730-1750 (2019).

127 Niu, P., Liu, G. \& Cheng, H.-M. Nitrogen Vacancy-Promoted Photocatalytic Activity of Graphitic Carbon Nitride. J. Phys. Chem. C 116, 11013-11018 (2012).

128 Niu, P., Yin, L.-C., Yang, Y.-Q., Liu, G. \& Cheng, H.-M. Increasing the Visible Light Absorption of Graphitic Carbon Nitride (Melon) Photocatalysts by Homogeneous Self-Modification with Nitrogen Vacancies. Adv. Mater. 26, 8046-8052 (2014).

129 Choudhury, B., Paul, K. K., Sanyal, D., Hazarika, A. \& Giri, P. K. Evolution of Nitrogen-Related Defects in Graphitic Carbon Nitride Nanosheets Probed by Positron Annihilation and Photoluminescence Spectroscopy. J. Phys. Chem. C 122, 9209-9219 (2018). 
130 Fang, Y. \& Wang, X. Photocatalytic $\mathrm{CO}_{2}$ conversion by polymeric carbon nitrides. Chem. Commun. 54, 5674-5687 (2018).

131 Xia, P. et al. Designing Defective Crystalline Carbon Nitride to Enable Selective $\mathrm{CO}_{2}$ Photoreduction in the Gas Phase. Adv. Funct. Mater. 29, 1900093 (2019).

$132 \mathrm{Wu}, \mathrm{H} .-\mathrm{Z}$. et al. Theoretical insight into the mechanism of photoreduction of $\mathrm{CO}_{2}$ to $\mathrm{CO}$ by graphitic carbon nitride. Phys. Chem. Chem. Phys. 21, 1514-1520 (2019).

133 Jiang, L. et al. Doping of graphitic carbon nitride for photocatalysis: A reveiw. Appl. Catal. B 217, 388-406 (2017).

134 Wang, Y., Li, H., Yao, J., Wang, X. \& Antonietti, M. Synthesis of boron doped polymeric carbon nitride solids and their use as metal-free catalysts for aliphatic $\mathrm{C}-\mathrm{H}$ bond oxidation. Chem. Sci. 2, 446-450 (2011).

135 Zhang, G. et al. Iodine Modified Carbon Nitride Semiconductors as Visible Light Photocatalysts for Hydrogen Evolution. Adv. Mater. 26, 805-809 (2014).

136 Yanagida, S., Kabumoto, A., Mizumoto, K., Pac, C. \& Yoshino, K. Poly(p-phenylene)-catalysed photoreduction of water to hydrogen. J. Chem. Soc. Chem. Commun., 474-475 (1985).

137 Matsuoka, S., Kohzuki, T., Nakamura, A., Pac, C. \& Yanagida, S. Efficient visible-light-driven photocatalysis. Poly(pyridine-2,5-diyl)-catalysed hydrogen photoevolution and photoreduction of carbonyl compounds. J. Chem. Soc. Chem. Commun., 580-581 (1991).

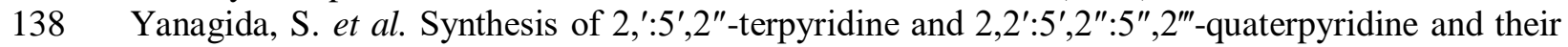
photocatalysis of the reduction of water. J. Chem. Soc., Perkin Trans. 2, 1963-1969 (1996).

139 Lee, J.-S. M. \& Cooper, A. I. Advances in Conjugated Microporous Polymers. Chem. Rev. 120, 2171-2214 (2020).

140 Dai, C. \& Liu, B. Conjugated polymers for visible-light-driven photocatalysis. Energy Environ. Sci. 13, 24-52 (2020).

141 Zhang, G., Lan, Z.-A. \& Wang, X. Conjugated Polymers: Catalysts for Photocatalytic Hydrogen Evolution. Angew. Chem. Int. Ed. 55, 15712-15727 (2016).

142 Woods, D. J., Sprick, R. S., Smith, C. L., Cowan, A. J. \& Cooper, A. I. A Solution-Processable Polymer Photocatalyst for Hydrogen Evolution from Water. Adv. Energy Mater. 7, 1700479 (2017).

143 Li, L., Lo, W.-y., Cai, Z., Zhang, N. \& Yu, L. Donor-Acceptor Porous Conjugated Polymers for Photocatalytic Hydrogen Production: The Importance of Acceptor Comonomer. Macromolecules 49, 6903-6909 (2016).

$144 \mathrm{Yu}, \mathrm{X}$. et al. Eosin Y-Functionalized Conjugated Organic Polymers for Visible-Light-Driven $\mathrm{CO}_{2}$ Reduction with $\mathrm{H}_{2} \mathrm{O}$ to $\mathrm{CO}$ with High Efficiency. Angew. Chem. Int. Ed. 58, 632-636 (2019).

145 Lan, Z.-A., Fang, Y., Zhang, Y. \& Wang, X. Photocatalytic Oxygen Evolution from Functional Triazine-Based Polymers with Tunable Band Structures. Angew. Chem. Int. Ed. 57, 470-474 (2018).

146 Wang, L. et al. Conjugated Microporous Polymer Nanosheets for Overall Water Splitting Using Visible Light. Adv. Mater. 29, 1702428 (2017).

147 Aitchison, C. M. et al. Synthetic approaches to artificial photosynthesis: general discussion. Faraday Discuss. 215, 242-281 (2019).

148 Sprick, R. S. et al. Nitrogen Containing Linear Poly(phenylene) Derivatives for Photo-catalytic Hydrogen Evolution from Water. Chem. Mater. 30, 5733-5742 (2018).

149 Wang, L. et al. Photocatalytic oxygen evolution from low-bandgap conjugated microporous polymer nanosheets: a combined first-principles calculation and experimental study. Nanoscale $\mathbf{9}$, 4090-4096 (2017).

150 Facchetti, A. $\pi$-Conjugated Polymers for Organic Electronics and Photovoltaic Cell Applications. Chem. Mater. 23, 733-758 (2011).

151 Sprick, R. S. et al. Maximising the hydrogen evolution activity in organic photocatalysts by copolymerisation. J. Mater. Chem. A 6, 11994-12003 (2018). 
152 Sprick, R. S. et al. Visible-Light-Driven Hydrogen Evolution Using Planarized Conjugated Polymer Photocatalysts. Angew. Chem. Int. Ed. 55, 1792-1796 (2016).

153 Zhao, Y. et al. Effect of Linking Pattern of Dibenzothiophene-S,S-dioxide-Containing Conjugated Microporous Polymers on the Photocatalytic Performance. Macromolecules 51, 9502-9508 (2018).

154 Schwarz, C. et al. Does Conjugation Help Exciton Dissociation? A Study on Poly(p-phenylene)s in Planar Heterojunctions with $\mathrm{C}_{60}$ or TNF. Adv. Mater. 24, 922-925 (2012).

155 Schwarz, C. et al. Role of the effective mass and interfacial dipoles on exciton dissociation in organic donor-acceptor solar cells. Phys. Rev. B 87, 155205 (2013).

156 Zhang, W. et al. Systematic Improvement in Charge Carrier Mobility of Air Stable Triarylamine Copolymers. J. Am. Chem. Soc. 131, 10814-10815 (2009).

157 Sprick, R. S. et al. Tunable Organic Photocatalysts for Visible-Light-Driven Hydrogen Evolution. J. Am. Chem. Soc. 137, 3265-3270 (2015).

Systematic and continuous control over the optical band gap of conjugated microporous polymers by statistical copolymerization.

158 Vyas, V. S. \& Lotsch, B. V. Organic polymers form fuel from water. Nature 521, 41-42 (2015).

159 Sprick, R. S. et al. Extended conjugated microporous polymers for photocatalytic hydrogen evolution from water. Chem. Commun. 52, 10008-10011 (2016).

$160 \mathrm{Xu}, \mathrm{Y}$. \& Jiang, D. Structural insights into the functional origin of conjugated microporous polymers: geometry-management of porosity and electronic properties. Chem. Commun. 50, 27812783 (2014).

161 Ritchie, J., Crayston, J. A., Markham, J. P. J. \& Samuel, I. D. W. Effect of meta-linkages on the photoluminescence and electroluminescence properties of light-emitting polyfluorene alternating copolymers. J. Mater. Chem. 16, 1651-1656 (2006).

162 Bruno, A., Reynolds, L. X., Dyer-Smith, C., Nelson, J. \& Haque, S. A. Determining the Exciton Diffusion Length in a Polyfluorene from Ultrafast Fluorescence Measurements of Polymer/Fullerene Blend Films. J. Phys. Chem. C 117, 19832-19838 (2013).

163 Sprick, R. S. et al. Photocatalytic Hydrogen Evolution from Water Using Fluorene and Dibenzothiophene Sulfone-Conjugated Microporous and Linear Polymers. Chem. Mater. 31, 305313 (2019).

164 Aitchison, Catherine M., Sprick, R. S. \& Cooper, A. I. Emulsion polymerization derived organic photocatalysts for improved light-driven hydrogen evolution. J. Mater. Chem. A 7, 2490-2496 (2019).

165 Noriega, R. et al. A general relationship between disorder, aggregation and charge transport in conjugated polymers. Nature Mater. 12, 1038-1044 (2013).

166 Podzorov, V. Long and winding polymeric roads. Nature Mater. 12, 947-948 (2013).

167 Kosco, J. et al. Enhanced photocatalytic hydrogen evolution from organic semiconductor heterojunction nanoparticles. Nature Mater. 19, 559-565 (2020).

168 Yang, H., Li, X., Sprick, R. S. \& Cooper, A. I. Conjugated polymer donor-molecular acceptor nanohybrids for photocatalytic hydrogen evolution. Chem. Commun. 56, 6790-6793 (2020).

169 Guiglion, P., Monti, A. \& Zwijnenburg, M. A. Validating a Density Functional Theory Approach for Predicting the Redox Potentials Associated with Charge Carriers and Excitons in Polymeric Photocatalysts. J. Phys. Chem. C 121, 1498-1506 (2017).

170 Few, S., Frost, J. M. \& Nelson, J. Models of charge pair generation in organic solar cells. Phys. Chem. Chem. Phys. 17, 2311-2325 (2015).

171 Bai, Y. et al. Accelerated Discovery of Organic Polymer Photocatalysts for Hydrogen Evolution from Water through the Integration of Experiment and Theory. J. Am. Chem. Soc. 141, 9063-9071 (2019).

Potential of machine learning and high-throughput screening in organic polymeric photocatalysis. 
172 Wilbraham, L., Berardo, E., Turcani, L., Jelfs, K. E. \& Zwijnenburg, M. A. High-Throughput Screening Approach for the Optoelectronic Properties of Conjugated Polymers. J. Chem. Inf. Model 58, 2450-2459 (2018).

173 Li, L. et al. Rational Design of Porous Conjugated Polymers and Roles of Residual Palladium for Photocatalytic Hydrogen Production. J. Am. Chem. Soc. 138, 7681-7686 (2016).

174 Pati, P. B. et al. An experimental and theoretical study of an efficient polymer nano-photocatalyst for hydrogen evolution. Energy Environ. Sci. 10, 1372-1376 (2017).

175 Kosco, J. et al. The Effect of Residual Palladium Catalyst Contamination on the Photocatalytic Hydrogen Evolution Activity of Conjugated Polymers. Adv. Energy Mater. 8, 1802181 (2018).

176 Kosco, J. \& McCulloch, I. Residual Pd Enables Photocatalytic $\mathrm{H}_{2}$ Evolution from Conjugated Polymers. ACS Energy Lett. 3, 2846-2850 (2018).

177 Liu, M., Guo, L., Jin, S. \& Tan, B. Covalent triazine frameworks: synthesis and applications. $J$. Mater. Chem. A 7, 5153-5172 (2019).

178 Kuhn, P., Antonietti, M. \& Thomas, A. Porous, Covalent Triazine-Based Frameworks Prepared by Ionothermal Synthesis. Angew. Chem. Int. Ed. 47, 3450-3453 (2008).

179 Bojdys, M. J., Jeromenok, J., Thomas, A. \& Antonietti, M. Rational Extension of the Family of Layered, Covalent, Triazine-Based Frameworks with Regular Porosity. Adv. Mater. 22, 2202-2205 (2010).

180 Kuhn, P., Thomas, A. \& Antonietti, M. Toward Tailorable Porous Organic Polymer Networks: A High-Temperature Dynamic Polymerization Scheme Based on Aromatic Nitriles. Macromolecules 42, 319-326 (2009).

181 Katekomol, P., Roeser, J., Bojdys, M., Weber, J. \& Thomas, A. Covalent Triazine Frameworks Prepared from 1,3,5-Tricyanobenzene. Chem. Mater. 25, 1542-1548 (2013).

182 Kuecken, S. et al. Fast tuning of covalent triazine frameworks for photocatalytic hydrogen evolution. Chem. Commun. 53, 5854-5857 (2017).

183 Kuecken, S., Schmidt, J., Zhi, L. \& Thomas, A. Conversion of amorphous polymer networks to covalent organic frameworks under ionothermal conditions: a facile synthesis route for covalent triazine frameworks. J. Mater. Chem. A 3, 24422-24427 (2015).

$184 \mathrm{Xie}$, J. et al. Efficient visible light-driven water oxidation and proton reduction by an ordered covalent triazine-based framework. Energy Environ. Sci. 11, 1617-1624 (2018).

185 Liu, M. et al. Crystalline Covalent Triazine Frameworks by In Situ Oxidation of Alcohols to Aldehyde Monomers. Angew. Chem. Int. Ed. 57, 11968-11972 (2018).

186 Zhang, S. et al. Strong-Base-Assisted Synthesis of a Crystalline Covalent Triazine Framework with High Hydrophilicity via Benzylamine Monomer for Photocatalytic Water Splitting. Angew. Chem. Int. Ed. 59, 6007-6014 (2020).

Crystalline covalent triazine framework synthesized by strong base assisted reaction between amidine and benzylamine-functionalized monomers for high photocatalytic hydrogen evolution activity and for overall water splitting.

187 Liu, J. et al. Solution Synthesis of Semiconducting Two-Dimensional Polymer via Trimerization of Carbonitrile. J. Am. Chem. Soc. 139, 11666-11669 (2017).

188 Liu, J., Lyu, P., Zhang, Y., Nachtigall, P. \& Xu, Y. New Layered Triazine Framework/Exfoliated 2D Polymer with Superior Sodium-Storage Properties. Adv. Mater. 30, 1705401 (2018).

189 Yadav, R. K., Kumar, A., Park, N.-J., Kong, K.-J. \& Baeg, J.-O. A highly efficient covalent organic framework film photocatalyst for selective solar fuel production from $\mathrm{CO}_{2}$. J. Mater. Chem. A 4, 9413-9418 (2016).

190 Guo, L. et al. Engineering heteroatoms with atomic precision in donor-acceptor covalent triazine frameworks to boost photocatalytic hydrogen production. J. Mater. Chem. A 6, 19775-19781 (2018).

191 Wang, Y., Chen, J., Wang, G., Li, Y. \& Wen, Z. Perfluorinated Covalent Triazine Framework Derived Hybrids for the Highly Selective Electroconversion of Carbon Dioxide into Methane. Angew. Chem. Int. Ed. 57, 13120-13124 (2018). 
192 Cheng, Z. et al. Efficient Visible-Light-Driven Photocatalytic Hydrogen Evolution on PhosphorusDoped Covalent Triazine-Based Frameworks. ACS Appl. Mater. Interfaces 10, 41415-41421 (2018).

193 Jiang, X., Wang, P. \& Zhao, J. 2D covalent triazine framework: a new class of organic photocatalyst for water splitting. J. Mater. Chem. A 3, 7750-7758 (2015).

194 Bi, J. et al. Covalent Triazine-Based Frameworks as Visible Light Photocatalysts for the Splitting of Water. Macromol. Rapid Commun. 36, 1799-1805 (2015).

195 Schwinghammer, K., Hug, S., Mesch, M. B., Senker, J. \& Lotsch, B. V. Phenyl-triazine oligomers for light-driven hydrogen evolution. Energy Environ. Sci. 8, 3345-3353 (2015).

Smaller phenyltriazine oligomers with higher number of unreacted nitrile moieties show improved photocatalytic activity compared to extended covalent triazine frameworks obtained via the high-temperature ionothermal route.

196 Wang, K. et al. Covalent Triazine Frameworks via a Low-Temperature Polycondensation Approach. Angew. Chem. Int. Ed. 56, 14149-14153 (2017).

197 Meier, C. B. et al. Structure-property relationships for covalent triazine-based frameworks: The effect of spacer length on photocatalytic hydrogen evolution from water. Polymer 126, 283-290 (2017).

198 Meier, C. B. et al. Structurally Diverse Covalent Triazine-Based Framework Materials for Photocatalytic Hydrogen Evolution from Water. Chem. Mater. 31, 8830-8838 (2019).

199 Côté, A. P. et al. Porous, Crystalline, Covalent Organic Frameworks. Science 310, 1166 (2005).

200 Rowan, S. J., Cantrill, S. J., Cousins, G. R. L., Sanders, J. K. M. \& Stoddart, J. F. Dynamic Covalent Chemistry. Angew. Chem. Int. Ed. 41, 898-952 (2002).

201 Jin, Y., Yu, C., Denman, R. J. \& Zhang, W. Recent advances in dynamic covalent chemistry. Chem. Soc. Rev. 42, 6634-6654 (2013).

202 Diercks, C. S. \& Yaghi, O. M. The atom, the molecule, and the covalent organic framework. Science 355, eaal1585 (2017).

203 Lohse, M. S. \& Bein, T. Covalent Organic Frameworks: Structures, Synthesis, and Applications. Adv. Funct. Mater. 28, 1705553 (2018).

204 Waller, P. J., Gándara, F. \& Yaghi, O. M. Chemistry of Covalent Organic Frameworks. Acc. Chem. Res. 48, 3053-3063 (2015).

205 Huang, N., Wang, P. \& Jiang, D. Covalent organic frameworks: a materials platform for structural and functional designs. Nat. Rev. Mater. 1, 16068 (2016).

206 Bisbey, R. P. \& Dichtel, W. R. Covalent Organic Frameworks as a Platform for Multidimensional Polymerization. ACS Central Sci. 3, 533-543 (2017).

207 Geng, K. et al. Covalent Organic Frameworks: Design, Synthesis, and Functions. Chem. Rev. (2020), DOI: 10.1021/acs.chemrev.9b00550.

208 Lyle, S. J., Waller, P. J. \& Yaghi, O. M. Covalent Organic Frameworks: Organic Chemistry Extended into Two and Three Dimensions. Trends Chem. 1, 172-184 (2019).

209 Pachfule, P. et al. Diacetylene Functionalized Covalent Organic Framework (COF) for Photocatalytic Hydrogen Generation. J. Am. Chem. Soc. 140, 1423-1427 (2018).

$210 \mathrm{Fu}$, Y. et al. Azine-based covalent organic frameworks as metal-free visible light photocatalysts for $\mathrm{CO}_{2}$ reduction with $\mathrm{H}_{2} \mathrm{O}$. Appl. Catal. B 239, 46-51 (2018).

211 Thote, J. et al. A Covalent Organic Framework-Cadmium Sulfide Hybrid as a Prototype Photocatalyst for Visible-Light-Driven Hydrogen Production. Chem. Eur. J. 20, 15961-15965 (2014).

212 Lei, K. et al. A Metal-Free Donor-Acceptor Covalent Organic Framework Photocatalyst for Visible-Light-Driven Reduction of $\mathrm{CO}_{2}$ with $\mathrm{H}_{2} \mathrm{O}$. ChemSusChem 13, 1725-1729 (2020).

213 He, T., Geng, K. \& Jiang, D. Engineering Covalent Organic Frameworks for Light-Driven Hydrogen Production from Water. ACS Mater. Lett. 1, 203-208 (2019).

214 Chen, J. et al. Synthesis of bipyridine-based covalent organic frameworks for visible-light-driven photocatalytic water oxidation. Appl. Catal. B 262, 118271 (2020). 
215 Stegbauer, L., Schwinghammer, K. \& Lotsch, B. V. A hydrazone-based covalent organic framework for photocatalytic hydrogen production. Chem. Sci. 5, 2789-2793 (2014).

216 Vyas, V. S. et al. A tunable azine covalent organic framework platform for visible light-induced hydrogen generation. Nat. Commun. 6, 8508 (2015).

Structural and (opto)electronic properties of COFs can be fine-tuned at the building block level to improve hydrogen evolution photocatalysis.

217 Haase, F., Banerjee, T., Savasci, G., Ochsenfeld, C. \& Lotsch, B. V. Structure-property-activity relationships in a pyridine containing azine-linked covalent organic framework for photocatalytic hydrogen evolution. Faraday Discuss. 201, 247-264 (2017).

218 Stegbauer, L. et al. Tailor-Made Photoconductive Pyrene-Based Covalent Organic Frameworks for Visible-Light Driven Hydrogen Generation. Adv. Energy Mater. 8, 1703278 (2018).

219 Wang, X. et al. Sulfone-containing covalent organic frameworks for photocatalytic hydrogen evolution from water. Nat. Chem. 10, 1180-1189 (2018).

Enhanced crystallinity and hydrophilicity of the COF backbone is beneficial for photocatalytic aqueous proton reduction.

220 Banerjee, T. \& Lotsch, B. V. The wetter the better. Nat. Chem. 10, 1175-1177 (2018).

221 DeBlase, C. R. \& Dichtel, W. R. Moving Beyond Boron: The Emergence of New Linkage Chemistries in Covalent Organic Frameworks. Macromolecules 49, 5297-5305 (2016).

222 Yaghi, O. M., Kalmutzki, M.J. \& Diercks, C.S. in Introduction to Reticular Chemistry (ed O.M. Yaghi, Kalmutzki, M.J. \& Diercks, C.S.) Ch. 8, 197-223 (Wiley-VCH Verlag GmbH \& Co. KGaA, 2019).

223 Segura, J. L., Mancheño, M. J. \& Zamora, F. Covalent organic frameworks based on Schiff-base chemistry: synthesis, properties and potential applications. Chem. Soc. Rev. 45, 5635-5671 (2016).

224 Li, H., Li, H., Dai, Q., Li, H. \& Brédas, J.-L. Hydrolytic Stability of Boronate Ester-Linked Covalent Organic Frameworks. Adv. Theory Simul. 1, 1700015 (2018).

225 Niu, W., O'Sullivan, C., Rambo, B. M., Smith, M. D. \& Lavigne, J. J. Self-repairing polymers: poly(dioxaborolane)s containing trigonal planar boron. Chem. Commun., 4342-4344 (2005).

226 Korich, A. L. \& Iovine, P. M. Boroxine chemistry and applications: A perspective. Dalton Trans. 39, 1423-1431 (2010).

227 Waller, P. J. et al. Chemical Conversion of Linkages in Covalent Organic Frameworks. J. Am. Chem. Soc. 138, 15519-15522 (2016).

$228 \mathrm{Li}, \mathrm{X}$. et al. Facile transformation of imine covalent organic frameworks into ultrastable crystalline porous aromatic frameworks. Nat. Commun. 9, 2998 (2018).

229 Han, X. et al. Chiral Covalent Organic Frameworks with High Chemical Stability for Heterogeneous Asymmetric Catalysis. J. Am. Chem. Soc. 139, 8693-8697 (2017).

230 Kandambeth, S. et al. Construction of Crystalline 2D Covalent Organic Frameworks with Remarkable Chemical (Acid/Base) Stability via a Combined Reversible and Irreversible Route. $J$. Am. Chem. Soc. 134, 19524-19527 (2012).

231 Biswal, B. P. et al. Mechanochemical Synthesis of Chemically Stable Isoreticular Covalent Organic Frameworks. J. Am. Chem. Soc. 135, 5328-5331 (2013).

232 Haase, F. et al. Topochemical conversion of an imine- into a thiazole-linked covalent organic framework enabling real structure analysis. Nat. Commun. 9, 2600 (2018).

233 Lyu, H., Diercks, C. S., Zhu, C. \& Yaghi, O. M. Porous Crystalline Olefin-Linked Covalent Organic Frameworks. J. Am. Chem. Soc. 141, 6848-6852 (2019).

234 Zhuang, X. et al. A two-dimensional conjugated polymer framework with fully sp ${ }^{2}$-bonded carbon skeleton. Polym. Chem. 7, 4176-4181 (2016).

235 Jin, E. et al. Two-dimensional $\mathrm{sp}^{2}$ carbon-conjugated covalent organic frameworks. Science 357, 673 (2017).

236 Jin, E. et al. Designed synthesis of stable light-emitting two-dimensional $\mathrm{sp}^{2}$ carbon-conjugated covalent organic frameworks. Nat. Commun. 9, 4143 (2018). 
237 Xu, J. et al. An Olefin-Linked Covalent Organic Framework as a Flexible Thin-Film Electrode for a High-Performance Micro-Supercapacitor. Angew. Chem. Int. Ed. 58, 12065-12069 (2019).

238 Jin, E. et al. 2D sp ${ }^{2}$ Carbon-Conjugated Covalent Organic Frameworks for Photocatalytic Hydrogen Production from Water. Chem 5, 1632-1647 (2019).

239 Jin, S. et al. Charge Dynamics in A Donor-Acceptor Covalent Organic Framework with Periodically Ordered Bicontinuous Heterojunctions. Angew. Chem. Int. Ed. 52, 2017-2021 (2013).

240 Jakowetz, A. C. et al. Excited-State Dynamics in Fully Conjugated 2D Covalent Organic Frameworks. J. Am. Chem. Soc. 141, 11565-11571 (2019).

241 Rogge, S. M. J. et al. Metal-organic and covalent organic frameworks as single-site catalysts. Chem. Soc. Rev. 46, 3134-3184 (2017).

242 Liang, H.-P. et al. Rhenium-Metalated Polypyridine-Based Porous Polycarbazoles for VisibleLight CO2 Photoreduction. ACS Catal. 9, 3959-3968 (2019).

243 Kasap, H. et al. Solar-Driven Reduction of Aqueous Protons Coupled to Selective Alcohol Oxidation with a Carbon Nitride-Molecular Ni Catalyst System. J. Am. Chem. Soc. 138, 91839192 (2016).

244 Caputo, C. A. et al. Photocatalytic Hydrogen Production using Polymeric Carbon Nitride with a Hydrogenase and a Bioinspired Synthetic Ni Catalyst. Angew. Chem. Int. Ed. 53, 11538-11542 (2014).

245 Luo, Z., Zhou, M. \& Wang, X. Cobalt-based cubane molecular co-catalysts for photocatalytic water oxidation by polymeric carbon nitrides. Appl. Catal. B 238, 664-671 (2018).

246 Kuriki, R. et al. Excited-State Dynamics of Graphitic Carbon Nitride Photocatalyst and Ultrafast Electron Injection to a Ru(II) Mononuclear Complex for Carbon Dioxide Reduction. J. Phys. Chem. C 122, 16795-16802 (2018).

247 Ma, B. et al. Efficient Visible-Light-Driven $\mathrm{CO}_{2}$ Reduction by a Cobalt Molecular Catalyst Covalently Linked to Mesoporous Carbon Nitride. J. Am. Chem. Soc. 142, 6188-6195 (2020).

$248 \mathrm{Fu}, \mathrm{Z}$. et al. A stable covalent organic framework for photocatalytic carbon dioxide reduction. Chem. Sci. 11, 543-550 (2020).

249 Yang, S. et al. 2D Covalent Organic Frameworks as Intrinsic Photocatalysts for Visible LightDriven $\mathrm{CO}_{2}$ Reduction. J. Am. Chem. Soc. 140, 14614-14618 (2018).

$\mathrm{CO}_{2}$ photoreduction to $\mathrm{CO}$ with high selectivity using a true single-site COF photocatalyst.

250 Banerjee, T. et al. Single-Site Photocatalytic $\mathrm{H}_{2}$ Evolution from Covalent Organic Frameworks with Molecular Cobaloxime Co-Catalysts. J. Am. Chem. Soc. 139, 16228-16234 (2017).

251 Biswal, B. P. et al. Sustained Solar $\mathrm{H}_{2}$ Evolution from a Thiazolo[5,4-d]thiazole-Bridged Covalent Organic Framework and Nickel-Thiolate Cluster in Water. J. Am. Chem. Soc. 141, 11082-11092 (2019).

252 Gottschling, K. et al. Rational Design of Covalent Cobaloxime-Covalent Organic Framework Hybrids for Enhanced Photocatalytic Hydrogen Evolution. J. Am. Chem. Soc. 142, 12146-12156 (2020).

253 Gao, G., Jiao, Y., Waclawik, E. R. \& Du, A. Single Atom (Pd/Pt) Supported on Graphitic Carbon Nitride as an Efficient Photocatalyst for Visible-Light Reduction of Carbon Dioxide. J. Am. Chem. Soc. 138, 6292-6297 (2016).

254 Takanabe, K. et al. Photocatalytic hydrogen evolution on dye-sensitized mesoporous carbon nitride photocatalyst with magnesium phthalocyanine. Phys. Chem. Chem. Phys. 12, 13020-13025 (2010).

255 Singh-Rachford, T. N. \& Castellano, F. N. Photon upconversion based on sensitized triplet-triplet annihilation. Coord. Chem. Rev. 254, 2560-2573 (2010).

256 Smith, M. B. \& Michl, J. Singlet Fission. Chem. Rev. 110, 6891-6936 (2010).

257 Li, X., Yu, J. \& Jaroniec, M. Hierarchical photocatalysts. Chem. Soc. Rev. 45, 2603-2636 (2016).

258 Banerjee, T. et al. Sub-stoichiometric 2D covalent organic frameworks from tri- and tetratopic linkers. Nat. Commun. 10, 2689 (2019).

259 Jiang, W. et al. Three-dimensional photocatalysts with a network structure. J. Mater. Chem. A 5, 5661-5679 (2017). 
260 Guan, X., Chen, F., Fang, Q. \& Qiu, S. Design and applications of three dimensional covalent organic frameworks. Chem. Soc. Rev. 49, 1357-1384 (2020).

261 Idriss, H. The elusive photocatalytic water splitting reaction using sunlight on suspended nanoparticles: is there a way forward? Catal. Sci. Technol. 10, 304-310 (2020).

262 Chen, S., Takata, T. \& Domen, K. Particulate photocatalysts for overall water splitting. Nat. Rev. Mater. 2, 17050 (2017).

263 Ferreira, K. N., Iverson, T. M., Maghlaoui, K., Barber, J. \& Iwata, S. Architecture of the Photosynthetic Oxygen-Evolving Center. Science 303, 1831 (2004).

264 Heath-Apostolopoulos, I., Wilbraham, L. \& Zwijnenburg, M. A. Computational high-throughput screening of polymeric photocatalysts: exploring the effect of composition, sequence isomerism and conformational degrees of freedom. Faraday Discuss. 215, 98-110 (2019).

265 Wilbraham, L., Sprick, R. S., Jelfs, K. E. \& Zwijnenburg, M. A. Mapping binary copolymer property space with neural networks. Chem. Sci. 10, 4973-4984 (2019). 